\author{
GAldi, Amalia \\ amgaldi@unisa.it \\ professor of medieval history (Università degli Studi di Salerno)
}

Santoro, Alfredo M.

masantor@unisa.it

resercher of medieval archeology (Università degli Studi di Salerno)

\title{
Le diocèse de Salerne au Moyen Âge: dynamiques historiques et organisation de l'espace*
}

\author{
CASO \\ - The Diocese of Salerne in the Middle Ages: Historical Dynamics \\ and Organisation of the Space -
}

Abstract In the middle ages, particularly between the $10^{\text {th }}-14^{\text {th }}$ centuries, the Diocese of Salerno was among the most important ones in southern Italy. Its earliest records date back to the late $5^{\text {th }}$ century and during the $10^{\text {th }}$ it was elevated to archdiocese; so the city of Salerno became the center of a large diocese, among the largest of Southern Italy. This essay reconstructs the history of diocese and its public symbols (like the cathedral), demonstrating consistency with that of other Italian dioceses but, at the same time, also highlighting some specificity resulting from more general historical dynamics of Salerno and the role it played in the southern part of Italy.

Keywords Diocese, Salerno, Lombards, Normans, Angevins, ecclesiastical heritage, ecclesiastical geography, southern Italy, Cathedral, sarcophagi.

DOI 10.14232/belv.2015.2.4 http://dx.doi.org/10.14232/belv.2015.2.4

Cikkre való hivatkozás / How to cite this article:

Galdi, Amalia - Santoro, Alfredo M. (2015): Le diocèse de Salerne au Moyen Âge : dynamiques historiques et organisation de l'espace. Belvedere Meridionale vol. 27. no. 2. 48-71. pp

ISSN 1419-0222 (print) ISSN 2064-5929 (online, pdf)

\footnotetext{
La première partie de cette étude (Les événements historiques entre le ve et XIV siècle) - p. 49-58. a été rédigée par Amalia Galdi, la seconde (Organisation de l'espace et références monumentales) - p. 58-66. par Alfredo M. Santoro, tandis que les prémisses et l'annexe bibliographique sont communes aux deux auteurs. En outre, dans son ensemble, cette analyse est le résultat d'une voie de recherche partagée. Traduction en français par Clarisse Thévenin et Alfredo M. Santoro.
} 


\section{Avant propos}

La recherche présentée ici faisait partie du projet GDRE ( Aux fondements de la modernité étatique en Europe, l'héritage des clercs médiévaux») et représente la première synthèse de réflexions plus générales sur des aspects du diocèse de Salerne au Moyen Âge, présentée à trois réunions différentes aux membres du projet. Il est donc proposé ici le premier résultat d'une recherche en cours, visant à étudier le diocèse de Salerne du $v^{\mathrm{e}}$ au $\mathrm{Xv}^{\mathrm{e}}$ siècle ainsi que les événements historiques qui y sont liés, et ceux des organismes chargés de sa direction. Cette étude s'effectue à travers une méthodologie interdisciplinaire analysant les espaces territoriaux et de juridiction, ainsi que les attestations matérielles et monumentales.

La recherche en cours commence par un corpus documentaire et historiographique relativement vaste - présent dans l'annexe bibliographique - qui, au cours des dernières années, n’a connu de mise à jour importante ni en ce qui concerne les sources, ni pour ce qui regarde l'interprétation, à l'égard de laquelle il faut également signaler une absence substantielle d'approches comparatives avec d'autres diocèses de la même époque. De même, les études précédentes ayant poursuivi une perspective interdisciplinaire attentive à la dynamique de l'organisation et de l'articulation de l'espace diocésain, en particulier aux éléments de l'héritage des monuments, sont assez limitées.

Dans les deux essais qui suivent, appuyés de références bibliographiques essentielles, sont proposées une brève histoire du diocèse pour délimiter l'objet de la recherche dans son évolution temporelle, qui sera préliminaire à une enquête plus approfondie, et une analyse des aspects liés au territoire et à la topographie du diocèse dans son ensemble ainsi qu'à certaines composantes matérielles significatives propres à la cathédrale de Salerne au bas Moyen Âge. La période choisie - susceptible d'être étendue par la suite - correspond à une phase comprise entre le $v^{e}$ et le début du $\mathrm{XIV}^{\mathrm{e}}$ siècle, c'est-à-dire des origines de la documentation sur le diocèse jusqu'aux attestations des Rationes Decimarum de Campanie, datant de 1308-1310, limite chronologique ad quem délibérément choisie puisque ce document, source d'ordre fiscal, offre une première vision de la géographie diocésaine de Salerne.

$$
\text { - A.G. et A.M.S. - }
$$

\section{Les événements historiques entre le $V^{e}$ et $X I V^{e}$ siècle}

À Salerne, comme dans d'autres villes de Campanie du haut Moyen Âge, la tradition locale a revendiqué une continuité épiscopale dès les premiers siècles du christianisme, mais les traces relatives, outre qu'incertaines et fragmentaires, remontent à une période bien lointaine de l'existence présumée des premiers évêques. La plus ancienne liste épiscopale salernitaine, d'ailleurs limitée à une succession de noms, se trouve dans le Diptycon ou Liber Vitae du Liber Confratrum de l'église de Saint Mathieu, dont les débuts de rédaction remontent aux années 1061-1083. ${ }^{1}$ Toutefois, les différences entre cette liste et les noms enregistrés dans les inscriptions dictées par l'archevêque Alfano ${ }^{\text {er }}$ (1058-1085), à l'occasion de la déposition des reliques des saints évêques salernitains dans la basilique inférieure de la cathédrale, ${ }^{2}$ témoignent de la confusion et de l'indétermination qui régnaient encore au $\mathrm{XI}^{\mathrm{e}}$ siècle.

Selon le Liber Confratrum, le premier évêque salernitain aurait été Bonoso, mais il n'existe pas d'ultérieures attestations de son épiscopat. ${ }^{3}$ En revanche, une autre hypothèse

1 Garufi 1922. 231. La partie la plus ancienne du code vient du Liber vitae ou Diptycon, «o da note marginali di qualche sacramentario, o di qualche altro libro liturgico ormai scomparso», (230).

2 Les inscriptions dictées par Alfano sont au nombre de six, dont cinq datées de 1081 et une de 1078. Pour le texte des épigraphes, cf. : CARUCCI 1974.

3 Sur les hypothèses avancées sur son épiscopat, cf. CRISCI 1976. 57. 
veut que la succession épiscopale ait débuté avec Quingesio, lequel aurait vécu au ve siècle à l'époque du pape Gelasio I ${ }^{\text {er }}{ }^{4}$ Le récit de l'auteur anonyme du Chronicon Salernitanum ${ }^{5}$ raconte que ses reliques et celles de Cirino furent transférées par l'évêque Bernardo (849-860 environ) de la localité de Faiano à une église salernitaine qui leur est consacrée. Il s'agit cependant d'une hypothèse basée uniquement sur des indices ${ }^{6}$ et elle demeure incertaine même si Cirino et Quingesio furent évêques de Salerne, bien que leurs noms, accompagnés des épithètes de pontifes et confesseurs, figurent sur deux des pierres tombales d'Alfano. ${ }^{7}$ En effet, leurs noms n'apparaissent pas dans le Liber Confratrum, et la même tradition liturgique salernitaine ne les a pas reconnus en tant qu'évêques locaux, même si elle leur a rendu un culte en qualité de confesseurs. ${ }^{8}$

Par conséquent, en accord avec le peu de fonds que nous possédons sur la ville tardoantique, ${ }^{9}$ les témoignages qui nous sont restés ne nous permettent pas de savoir avec précision quand un siège épiscopal a été créé à Salerne. Il est toutefois possible que le diocèse ait été érigé avant la fin du v $v^{\mathrm{e}}$ siècle, c'est-à-dire précédemment à la période d'où remontent les informations attestées de son existence, et peut-être pas au hasard, quelques décennies suivant les interventions réalisées suite aux graves inondations qui touchèrent la ville entre la fin $\mathrm{du} \mathrm{IV}^{\mathrm{e}}$ et le début du $\mathrm{v}^{\mathrm{e}}$ siècle. C'est également à la même période où est attestée une réhabilitation des espaces thermaux retrouvés dans le complexe

4 P.F.Kher(Kher 1935.344.), suppose que le Quingesio à qui s'adresse le pape Gélase entre 494/495-496, un Campanien, soit à identifier avec un évêque salernitain.

5 Westerbergh 1956, chapitres 97, 98.

6 Comme le démontre Crisci 1976. 71-72.

7 CRISCI 1976. 71.

8 GAldi 2000. 102-103.

9 Sur la période romaine de la ville, avec références aux sources et bibliographie, cf. : AмARotтA 1989 et Romito 2013. 21-31. de San Pietro a Corte. ${ }^{10}$ Le $1^{\mathrm{er}}$ mars 499 en effet, un évêque du nom de Gaudenzio, ignoré du Liber Confratrum et des épigraphes d'Alfano, participe au premier synode général, célébré en la basilique romaine de Saint Pierre par le pape Symmaque. ${ }^{11}$

Des $\mathrm{v}^{\mathrm{e}}-\mathrm{vI}^{\mathrm{e}}$ siècles il y a aussi Grammazio, le premier évêque à recevoir un culte local, comme l'atteste une église qui lui est consacrée et mentionnée en $1026^{12}$ ainsi qu'une tradition hagiographique le démontre dont la première version écrite, en douze lectiones, est incluse en un code liturgique daté cependant de $1434 .{ }^{13}$ Son existence est confirmée par une inscription sur marbre placée au-dessus de l'urne de bois contenant les reliques attribuées à Grammazio, retrouvées suite à la démolition de l'autel de l'église consacrée au saint, et ordonnée par l'archevêque Gregorio Carafa (1664-1675). L'inscription a été perdue, mais il en reste la transcription du notaire Matteo Alfano sur la base de laquelle a été datée la déposition du corps au 25 janvier 490. Une autre hypothèse propose l'année 525, après que le prélat vixit in pace XLI annos, indications à reporter à la durée de son épiscopat, particulièrement long dans ce cas, ou aux années de son existence. ${ }^{14}$

Aucune information historique en revanche n'est disponible pour Vero et Valen-

10 Voir Peduto - Fiorillo - CoRolla 2013. Sur la récupération des structures thermales et la réalisation d'un espace funéraire, dans lequel se sont succédées des sépultures de 497 aux premières décennies du VII ${ }^{\mathrm{e}}$ siècle, cf. : LAMBERT 2013.

11 Il signe avec le titre Gaudentius episcopus ecclesiae Salernitanae, cf. : CRISCI 1976. 68.

12 Voir: Morcaldi - Schiani - De Stefano 1878. Nr. 787,126 . Sur la localisation de l'édifice et ses faits historiques, cf. : AMAROTTA - IANNELLI 1990, GALDI 2002-2003. 15-17.

13 Vita s. Gramatii episcopi salernitani, édité dans GALDI 2002-2003. 24-26. Le texte est anonyme et ne contient pas d'éléments pour reconstituer la biographie et l'épiscopat de Grammazio, se limitant à topoi traditionnellement hagiographiques apte à décrire le personnage du saint évêque (ibid., 20-23).

14 Galdi 2002-2003. 17-18. 
tiniano, attestés par la tradition locale parmi les évêques saints des premiers siècles, ${ }^{15}$ tandis qu'Eusterio (ou Austerio) souscrit en 536 les actes du V $V^{e}$ Synode de Constantinople, avec d'autres légats du pape Agapite $\mathrm{I}^{\mathrm{er}}$, signe d'une position de prestige pour le Saint Siège, ${ }^{16}$ et c'est encore à lui que s'adresse le pape Pélage, entre 555 et 560 , pour qu'il consacre l'oratoire érigé de l'abbé Vindimio en l'honneur des saints Crisante et Daria à l'intérieur du monastère fondé par le même abbé près des remparts de Salerne. ${ }^{17}$

L'absence d'autres attestations sur le diocèse pour les décennies suivant l'épiscopat d'Eusterio, jusqu'à la moitié du viI ${ }^{\mathrm{e}}$ siècle, quand les lombards de Bénévent soustrayèrent Salerne aux Byzantins (640 environ), correspond au silence plus général des sources écrites sur la ville pour les $\mathrm{VI}^{\mathrm{e}}-\mathrm{VII}{ }^{\mathrm{e}}$ siècles. ${ }^{18} \mathrm{Il}$ est fort peu probable que durant ces années le siège ait été occupé par Gaudioso, un évêque cité dans le Liber Confratrum, et qui selon une certaine tradition hagiographique tardive aurait été originaire de Naples. Distingué par ses dons d'humilité, de charité et de pauvreté, il aurait effectué différents miracles de son vivant et après sa mort, et aurait apporté la paix entre les Salernitains et les habitants de Bénévent; ce dernier fait, en particulier, laisserait supposer un épiscopat durant l'occupation lombarde de Salerne. Au caractère légendaire des sources hagiographiques sur Gaudioso, s'ajoute la probable confusion déjà observée par Hyppolite Deleheye en 1955, entre le présumé évêque salernitain et l'homonyme évêque africain vénéré comme un saint. ${ }^{19}$

15 Voir: Morcaldi - Schiani - De STefano 1878. nr. 787, 126. Sur la localisation de l'édifice et ses faits historiques cf. : AMAROTTA - IANNELLI 1990 ; GALDI 2002-2003. 15-17. ; CRISCI 1976. 65-67, où l'on propose aussi l'identification de Vero avec l'Ursus cité dans Liber Confratrum.

16 KeHR 1935. 340. 344.

17 KeHr 1935. 344.

18 Peduto 2013. 1.

19 Pour sources et bibliographie sur Gaudioso, cf. :
Après un évêque du nom de Luminoso, présent au synode romain de 649 , un long silence des sources écrites caractérise la période allant jusqu'à l'épiscopat de Rodoberto, ${ }^{20}$ membre de la délégation des évêques de Bénévent envoyée par le duc-prince Arigis II (758-787) à Charlemagne présent à ce moment-là à Capoue, et qui selon le récit de l'auteur anonyme du Chronicon Salernitanum, fut le premier à s'adresser directement au roi des Francs pour intercéder en faveur de Bénévent. ${ }^{21}$ En effet, de la principauté d'Arigis jusqu'en 974 environ, la plupart des informations sur les évêques salernitains est fournie par le chroniqueur. ${ }^{22} \mathrm{Ce}$ dernier, actif durant la principauté de Pandolfo Capodiferro (943-981), rédigeait la source la plus importante pour la reconstitution des évènements de la Langobardia méridionale, née par la suite de la chute du royaume lombard (774) précipitée par Charlemagne. Toutefois, les informations qu'il a fournies, à quelques exceptions près, ne sont pas suffisantes pour reconstituer le rôle effectué par chacun des prélats dans les évènements historiques de la ville, et ce n'est que rarement où il nous informe de leurs origines géographiques et sociales. Dans de nombreux cas, il se limite à une liste de noms, ce qui suggère le peu d'incidence du diocèse et de ses représentants dans le tumultueux cadre

CRISCI 1976. 84-85, ainsi que GALDi 2000. 110-112.

20 CRISCi 1976. 88-93. Le Liber Confratrum, cependant, énumère après Eusterio et avant Rodoberto, omettant Luminoso, Giovanni et Leone, les évêques Valerio, Gaudioso, Zaccaria, Colombo, Lupo, Renobato, Benedetto, Talarico, Audemario (GARUfi 1922. 231).

21 Westerbergh. 1956., chapitre 11. 45. Sur Rodoperto, cf. : CRISCI 1974. 95-97.

22 Douze évêques sont attestés dans le Chronicon (WEsTERBERGH. 1956) jusqu'à la création du siège métropolitique (983 environ), auxquels vont s'ajouter trois prélats nommés Pietro non cités par le chroniqueur (cf. : Taviani Carozzi 1991. 636-637) et peut-être Nicola, prédécesseur de Radoaldo, protagoniste du récit de la translatio des reliques de Vito, Modesto et Crescenza à l'embouchure du fleuve Sele à Polignano, dans les Pouilles (Crisci 1974. 98-99). 
politique qui caractérisait Salerne, ainsi que l'entière Campanie, dans les derniers siècles du haut Moyen Âge. Et son récit ne s'attarde pas non plus sur le respect ou non des canons durant les élections épiscopales - qui dans certains cas, comme nous le verront, sont conditionnées par le pouvoir politique - nonobstant l'utilisation des termes ordinarunt, sublimarunt, elegerunt interprétée comme l'expression d'un assentiment unanime des Salernitains. ${ }^{23}$ D'autre part, le contrôle exercé par les princes sur l'Église et la présence massive des églises privées, avec un clergé fondamentalement indépendant du pouvoir épiscopal - en particulier dans les fondations princières - devaient notoirement entraver l'activité pastorale des évêques. ${ }^{24}$ Lesquels, comme dans le reste des territoires lombards, étaient en outre soumis à un rare contrôle de la part du Saint Siège, incapable - ou peu intéressé - à faire respecter les normes canoniques. Une lettre de 874 du pape Jean VIII adressée au clergé de Salerne, pour une sentence émise par l'évêque Rachenaldo contra canones, et une intervention répétée de Jean VIII, de 874 à 878 , contre l'évêque Pietro en raison d'une procédure élective ${ }^{25}$ non canonique, font exception.

À la période d'Arigis, remonte également la première information sur la cathédrale de Salerne, réalisée à une époque imprécise mais vraisemblablement présente depuis le $\mathrm{v}^{\mathrm{e}}$ siècle au moins, quand les premiers évêques sont attestés. C'est le même chroniqueur qui nous informe qu'Arigis fut inhumé dans l'Ecclesia Dei Genitricis, ${ }^{26}$ de laquelle le peu de sources disponibles ne précisent pas l'emplacement exact. Cependant, d'après un document daté de novembre 1064, il a été supposé qu'elle se situait à l'est de l'archevêché, tandis que d'autres documents datés de 971 à mars 1058 laissent entendre que l'archevêché surgissait

\footnotetext{
23 Taviani Carozzi 1991. 643-644.

24 Taviani CarozZI 1991. 662-667.

25 Taviani Carozzi 1991. 638-639, 648-649.

26 Westerbergh 1956. Chapitre 17. 22.
}

sur la partie occidentale de la zone occupée actuellement et que l'Ecclesia maior lombarde était située à l'angle nord-est de la même zone. ${ }^{27}$

Pour reprendre la chronologie de la succession épiscopale, le chroniqueur nous informe que certains évêques régirent le diocèse à la moitié du $\mathrm{IX}^{\mathrm{e}}$ siècle, pour certains desquels il nous donne seulement le nom : Radoaldo, Pietro - ce dernier s'est enfui de Canosa pour Salerne après la conquête des Sarrazins, et n'a peut-être jamais revêtu le titre épiscopal, ${ }^{28}$ même s'il a doté la ville d'une église consacrée à Jean-Baptiste - ou bien Ratulo, Magnaldo, Teupo, Aione et Landemario. Pour ces deux derniers, il nous donne quelques détails supplémentaires, car le premier fut un homme illustre, bon et défenseur de l'Église, constructeur d'un ambon d'une grande beauté, tandis que le second était originaire de Nocera, non loin de Salerne. Son successeur fut Bernardo (849-860 environ), originaire de Latiniano, de bonne ascendance sociale. À son propos, le récit du chroniqueur se fait circonstancié et l'évêque se distingue des autres prélats susnommés car il était tenace défenseur de la liberté de l'Église par rapport au pouvoir politique, infatigable édificateur d'églises et initiateur de divers transferts de reliques. ${ }^{29}$

Ces translations, qui doteront Salerne de sacrés pignora auxquels était confié le soin de pourvoir à la salus publique et privée, ne fut pas une exclusivité de Bernardo car à la même période le phénomène fut particulièrement répandu en Campanie, parallèlement à avec un renouvellement du rôle de l'épis$\operatorname{copat}^{30}$; et le lien entre des évènements de ce genre et l'expression d'une identité citadine

27 CRISCI 2001. I. 26-27.

28 L'Anonyme fait prononcer à Pietro les paroles suivantes quand lui fut proposé de succéder à Radoaldo : «Ad regendam ecclesiam preesse me dico, in sedem ipsius ecclesie minime ascensurus sum» (chapitre 97, p. 97).

29 Westerbergh. 1956., chapitre 97-98., p. 97-99.

30 Voir: Cilento 1969. 689, 705-708 et Vitolo 1999. 95 ss. 
qui trouvait dans le sacré des contenus et des représentations, ${ }^{31}$ est désormais bien clair à l'historiographe. Toutefois, Bernardo pour la première fois à Salerne, conçut un programme cohérent de sacralizzazione de la ville sur le strict plan de la dévotion, mais aussi sur celui de la construction, regardant même le nouveau quartier salernitain Plaium Montis, où il édifia l'église du Sauveur, donnant ainsi une impulsion significative à son urbanisation. ${ }^{32}$ De manière considérable, les actions de l'évêque s'effectuent dans les années qui succèdent immédiatement à la formation de la Principauté de Salerne en 849, dans une phase durant laquelle l'autonomie de Salerne sur Bénévent créait les conditions idéales pour la ville et son Église pour construire ou renforcer sa propre conscience civile. ${ }^{33}$

Mais les qualités de Bernardo se mesurent aussi dans ses rapports avec le pouvoir princier d'une part, et avec la Curie romaine de l'autre. ${ }^{34}$ Celui-ci, lors de circonstances délicates impliquant les rapports ambigus qu'entretenaient les élites de Campanie avec la composante sarrazine, fut capable de s'opposer au pouvoir politique, jusqu'à quitter Salerne et se transférer à Rome, où il fut bien accueilli par le pape, et de revenir en ville uniquement quand on lui promit la construction d'une nouvelle résidence, après que la sienne ait été occupée par un ambassadeur des Sarrazins. ${ }^{35}$ Une indépendance certes non partagée par ses successeurs : Pierre II (861-862), proclamé élu par son père, le prince Ademario (856-861), et Rachenaldo (863-872 environ), preordinatus du prince Guaiferio $(861-880)^{36}$; et un rapport avec les pontifs romains qui ne se renouvellera pas, par

31 Voir, avec références aux sources et bibliographie, GALDI 2003. 1431-1433.

32 GALDI 2003. 1434-1435.

33 GALdi 2003. 1434-1435, 1440.

34 Sur les rapports entre la Papauté et les territoires lombards dans la seconde moitié du $\mathrm{IX}^{\mathrm{e}}$ siècle, $\mathrm{cf}$ : : GALDI 2003. 1437-1438.

35 Westerbergh. 1956., chapitre 99, 99-100.

36 Westerbergh. 1956., chapitre 100-101., 101-102., chapitre 105., 105. exemple avec Pierre III qui fut excommunié plusieurs fois par le pape Étienne V (885-891) parce qu'il avait refusé de se rendre à Rome pour la consécration. ${ }^{37}$

Sur les évêques du $\mathrm{x}^{\mathrm{e}}$ siècle, le chroniqueur est plus avare d'informations, un fait qui a été vu comme une réprobation de l'Anonyme envers l'Église salernitaine de cette période, par rapport à l'époque précédente dans laquelle semble régner une meilleure organisation diocésaine et une meilleure préoccupation pastorale. ${ }^{38}$ Et pourtant ce siècle, et en particulier la seconde moitié, a été parcouru d'évènements qui auront de grandes conséquences sur l'histoire future de la ville et de son diocèse. Cependant, les années allant de 936 à 949/950 sont marquées par l'épiscopat de Pierre (IV), figure éminente sur le plan pastoral, attentif à l'administration du clergé et de l'église locale, et surtout capable d'attirer les donations du prince, alors Gisulfo I ${ }^{\text {er }}$, qui aurait accru le patrimoine de l'épiscopat. ${ }^{39}$ Mais l'évènement le plus important remonte à 954, quand furent retrouvées les reliques de l'apôtre Mathieu in finibus Lucanie et transférées à Salerne, au temps de l'évêque Bernardo II. Le chroniqueur salernitain assista à l'évènement mais il est probable qu'il ne donna pas suite à son intention de narrer les circonstances de cette découverte ni aux prodiges qui l'accompagnèrent. ${ }^{40}$ La possession du corps, sinon immédiatement mais à plus ou moins long terme, contribua indubitablement à relever le prestige de la ville, contribuant à lui conférer un rôle politique de premier plan destiné à s'accroître au siècle suivant, et qui conduira à l'épithète de Opulenta Salernum dont Salerne usera sur ses

37 KeHr 1935. 345., nr. 8., 9, mais voir aussi nr. 7.

38 Taviani Carozzi 1991. 643. Au Ix ${ }^{\mathrm{e}}$ siècle remontent entre autres, les actes d'un concile postérieur à 849 , peut-être tenu à Bénévent, centré surtout sur la discipline des clercs et des laïcs. Taviani Carozzi 1991. 653-654.

39 KeHr 1935. 345, nr. 8., 9, mais voir aussi nr. 7.

40 Westerbergh. 1956., chapitre 165, 170. 
monnaies battues par les derniers lombards. ${ }^{41}$

Les années suivantes furent marquées par l'épiscopat de Pierre (VI) qui, précise le chroniqueur, était si cher au prince Gisulfo qu'il fut promu évêque par ce dernier ; un personnage tellement estimé par l'Anonyme - qui, bien entendu, retenait totalement légitime qu'un homme si fortement lié au pouvoir politique puisse gagner le siège épiscopal - qu'il promit d'en reparler plus longuement plus avant, intention à laquelle il ne donna pas suite. Pierre était en outre un habile médecin ${ }^{42}$ : c'était la première fois que l'exercice de cette profession par un évêque était mentionnée, d'autant plus que le Chronicon n'avait jamais fait référence à la médecine salernitaine, dont la renommée avait déjà dépassé amplement les frontières de la ville au cours de cette même période. ${ }^{43}$

Bientôt, le récit du chroniqueur aura pris fin sans que celui-ci ait pu assister à un autre évènement historique : l'élévation du diocèse à celui de siège métropolitain aux environs de 983, avec l'archevêque Amato (982-992). C'est une reconnaissance qui, dans les mêmes années, a également intéressé, sous différentes formes, les zones plus ou moins soumises au contrôle byzantin. Mais surtout - pour ce qui nous intéresse ici - elle impliqua (avant Salerne) Capoue (966) et Bénévent (969) ; tant et si bien que les diocèses lombards furent substantiellement réorganisés, décomposant le pouvoir primatial traditionnel du pape sur eux, un processus dans lequel entraient en jeu divers intérêts, politiques et ecclésiastiques, locaux et généraux, ainsi que l'historiogra-

41 Sur la translation et le culte de saint Mathieu, cf. : GALDI 1996; GALDI 1999.

42 Westerbergh. 1956., chapitre 163, 169.

43 Il y a peu d'informations disponibles sur la médecine salernitaine du haut Moyen Âge, sur ses origines, ce qui contredit l'extraordinaire développement qui la caractérisera à partir du $\mathrm{XI}^{\mathrm{e}}$ siècle. Parmi les nombreuses recherches sur la «Scuola » de médecine on signale, pour un status quaestionis sur les sources et l'historiographie: JACQUART - PARAVICINI BAGLIANI 2007. phie $^{44}$ l'a si bien expliqué. Le réseau diocésain cependant, était destiné à une réorganisation au cours du XI ${ }^{\mathrm{e}}$ siècle, dans lequel se sont mesurés également des intérêts généraux et particuliers, mêlés à des processus de plus grande envergure tel que le mouvement de Réforme de l'Église, et surtout l'installation normande qui aura profondément changé le cadre politique et institutionnel du sud de l'Italie, pesant même sur les compétences juridictionnelles ecclésiastiques. ${ }^{45}$

Pour Salerne, comme ailleurs, les conditions locales spécifiques ne furent pas étrangères aux mesures prises par le pape, auxquelles le rôle des évêques a peut-être contribué $^{46}$; toutefois, certaines différences ont été relevées entre les bulles papales adressées aux évêques de Salerne et de Bénévent, traduisant le contexte politique différent des deux villes et le rôle qu'elles revêtaient pour la Papauté, de plus en plus intéressée à partir de la moitié du $\mathrm{XI}^{\mathrm{e}}$ siècle par le contrôle des élections épiscopales salernitaines, ${ }^{47}$ encore conditionnées jusqu'à cette période, par l'intervention des princes. ${ }^{48}$

Les confins géographiques de la circonscription ecclésiastique, comme pour le diocèse de Bénévent, ne coïncidaient pas avec le domaine politique des princes de Salerne mais incluaient également une partie de l'actuelle Calabre et Basilicate, de façon à englober des sièges dépendants de Byzance, du moins formellement, comme on peut le noter dans la première bulle qui nous est parvenue, émise par Jean XV en 989, celle de 983 ayant été per-

44 De la vaste bibliographie cf. au moins les essais de Cosimo Damiano Fonseca, (Fonseca 1996) et Giuseppe Spinelli (SPINELli 1996).

45 Il s'agit des problématiques complexes et avec de nombreuses différences locales. Pour un cadre d'ensemble avec références bibliographiques, cf. : GALDI 2004a. 96-99.

46 Par exemple, le prédécesseur d'Amato, Jean (977-982) fut datarius et substitut-bibliothécaire du siège apostolique. CRISCI 1976. 147.

47 Taviani Carozzi 1991. 998-1008.

48 Taviani CarozZI 1991. 674. 
due. ${ }^{49}$ Les diocèses suffragants furent confirmés - dans une bulle de Léon IX sur laquelle cependant pèse un doute de falsification ${ }^{50}$ - à l'archevêque Jean en 1051, excepté Malvito, ${ }^{51}$ qui réapparaît dans la bulle d'Étienne IX de $1058^{52}$.

C'est justement en 1058 que débute l'activité épiscopale d'un des personnages les plus significatifs de l'histoire de l'Italie du Sud, l'archevêque Alfano (1058-1085), ${ }^{53}$ après une succession de prélats caractérisés par des épiscopats généralement brefs, bien que jalonnés de moments significatifs ${ }^{54}$.

Né entre 1015 et 1020 d'une famille noble, Alfano devint moine au Mont Cassin, et, comme on peut le lire dans le Chronicon Casinense, il fut eruditissimus dans les Saintes Écritures et hagiographe, prudentissimus et nobilissimus, ami de Desiderius, abbé de Mont Cassin, habile dans le chant et la médecine, abbé du monastère San Benedetto de Salerne, ${ }^{55}$ et enfin archevêque de la même ville. Le 24 mars 1058, Étienne IX lui confirma les droits dont jouissait déjà le diocèse salernitain, (en y ajoutant la juridiction sur Marturano et Cassano) et lui concéda la faculté ordinandi episcopos in aliis locis Salernitanae diocesis. ${ }^{56}$ Alfano se servit de celle-ci pour ériger trois nouveaux diocèses : Nusco, Acerno, et Sarno. À la même période, il a été observé que dans les bulles papales on commence à faire explicitement allusion au patrimoine du diocèse de Salerne, non fortuitement d'ailleurs, simulta-

49 Kenr 1935. 346, nr. 11.: ont été élus Paestum, Conza, Acerenza, Nola, Bisignano, Malvito, Cosenza.

50 Voir la récente édition avec le commentaire correspondant d'Anna Giordano (GIORDANO 2014. 50-53).

51 Voir: GALDI 2004a. 95-96, 100.

52 Kehr 1935. 350, nr. 21

53 Sur le personnage d'Alfano et son activité littéraire et scientifique, l'historiographie est abondante mais plutôt ancienne. Il est toujours utile de voir ACOCELLA 1958 et 1959.

54 CRISCI 1976. 159-186.

55 Hoffmann 1980. 355, 368, 411.

56 Kehr 1935. 350, nr. 21 nément à l'affirmation de la Réforme de l'Église, surtout à partir d'une bulle d'Alexandre II de 1067. ${ }^{57}$ Toutefois, bien que la reconstitution $\mathrm{du}$ patrimoine de ce siège ne fasse pas ici l'objet d'attention particulière, il est opportun de signaler au moins que des doutes ont été soulevés quant à l'authenticité de cette bulle, dans laquelle tous les anciens privilèges de l'Église salernitaine sont confirmés. ${ }^{58}$

Quoi qu'il en soit, l'activité d'Alfano coïncida avec une phase particulièrement complexe de l'histoire de la Principauté, marquée surtout par le gouvernement de Guaimario IV ( $\uparrow 3$ juin $1052)^{59}$ et que l'on peut difficilement résumer en raison de l'ampleur des évènements et de l'implication de Salerne dans de vastes et dynamiques affaires politiques, qui engagaient les principaux acteurs politiques de l'époque, des seigneurs Lombards aux empereurs d'Occident et d'Orient, à d'autres puissances méridionales, à la papauté, aux grandes abbayes méridionales, in primis Mont Cassin.

Alfano vécut donc un moment particulièrement délicat pour la ville, assista à son extension politique maximale et à son apogée en tant que capitale lombarde; mais aussi à sa crise et au passage - bien évidemment douloureux - aux Normands de Robert Guiscard qui conquirent Salerne en 1076.

S'ouvre ainsi une nouvelle ère pour Salerne, sous le signe d'une collaboration entre le Guiscard et Alfano si, comme il semblerait, la nouvelle cathédrale a été conçue et réalisée avec le concours de chacun des deux. Il est sûr que le Normand était conscient de ce que signifiait doter la ville d'une église grandiose consacrée à Saint Mathieu, qui soit à la fois un hommage au saint, à ses fidèles et à l'Église, mais surtout un extraordinaire témoignage de son pouvoir à livrer à l'histoire. ${ }^{60}$ Alfano devait

57 Taviani Carozzi 1991. 998-999.

58 GIORDANO 2014. 70-73.

59 Sur la principauté de Guaimario, cf. : TAviani CAROZZI 1991. 401-404, 843-847.

60 GALDI 1996. 70. 
aussitôt comprendre combien la communauté salernitaine avait, en un moment si délicat de son histoire, la nécessité de reconstruire et de réaffirmer son identité à travers de nouvelles formes politiques et culturelles. Durant la construction de l'édifice et la démolition des structures de l'ancienne cathédrale en 1080, on retrouva le sépulcre de saint Mathieu, un évènement salué avec joie par le pape Grégoire VII, qui se félicita avec Alfano, par qui il avait été informé. ${ }^{61}$ Selon la tradition, ce sera ensuite le même pape, exilé à Salerne, qui consacra la nouvelle Ecclesia maior. ${ }^{62}$

À partir de ce moment, le culte de l'apôtre connaîtra un grand développement, jusqu'à s'étendre même au-delà de la province ecclésiastique salernitaine, et en cela la ville continuera à trouver les raisons de sa cohésion et de son identité. ${ }^{63}$ La construction de la cathédrale, cependant, n'épuise pas le rôle d'Alfano sur le plan de la dévotion, puisque celui-ci compose des chants à caractère hagiographique pour Saint Mathieu et les principaux saints de la ville, dont certains furent introduits dans la liturgie salernitaine. De plus, Alfano prend soin de la déposition des corps des saints évêques salernitains dans la cathédrale, comme il a été dit précédemment. ${ }^{64}$

Le $\mathrm{XI}^{\mathrm{e}}$ siècle se terminera avec un autre privilège pour le diocèse salernitain, car Urbain II, le 20 juillet 1098, concéda à Alfano II la dignité de la primatie sur les sièges épiscopaux d'Acerenza et Conza avec les diocèses respectifs. ${ }^{65}$ Entre la fin du XI ${ }^{\mathrm{e}}$ et la moitié du XII ${ }^{\mathrm{e}}$ siècle, l'extension de la province ecclésiastique subira une contraction, avec la soustraction des sièges diocésains plus périphériques ${ }^{66}$; mais

61 Caspar 1923. Nr. VIII:8., p. 526-527. Sur la signification de cet évènement cf. : GALDI 2004a. 279-280.

62 Sur la zone dans laquelle fut érigée la cathédrale et pour une synthèse de son histoire architecturale cf. Crisci 1991. 29-51.

63 GALDI 2004a. 283-286.

64 Galdi 2004a. 281-283.

65 Sur la primatie, cf. : SANGERmano 2000. 77-94.

66 Kehr 1935. 341 ; cf. aussi: 258, nr. 45. malgré cela la période normande représentera une phase de forte croissance pour Salerne, économique et commerciale, mais aussi démographique et urbanistique. ${ }^{67}$

Il est certain que le personnage le plus représentatif du $\mathrm{XI}^{\mathrm{e}}$ siècle salernitain fut Romualdo Guarna (1152/53-1181), dont l'épiscopat fait suite à la longue direction du diocèse par Alfano II (1085/86-1121), non sans contrastes avec Ruggiero, fils et successeur du Guiscard à la tête du duché des Pouilles et de la Calabre, à celle de Romualdo Ir (1121-1136), qui s'est déclaré en faveur de l'antipape Anaclet II, appuyé en cette période par Ruggiero II, le premier souverain du Royaume de Sicile, et enfin de Guillaume de Ravenne (1137-1152), dont le nom est surtout lié à la construction du campanile roman de la cathédrale. ${ }^{68}$

Appartenant à une des familles salernitaines les plus prestigieuses, ${ }^{69}$ Romualdo fut protagoniste d'une activité culturelle et politique dépassant largement sa charge d'évêque de Salerne. Ainsi que l'on sait, il fut l'auteur d'un Chronicon, a été un personnage important à la cour sicilienne du roi Guillaume $\mathrm{I}^{\mathrm{er}}$, dont il fut aussi le médecin personnel, et qui enfin tint un rôle de premier ordre dans la paix de Venise (1177), à la fin des affrontements entre les «Comuni » et Frédéric I ${ }^{\text {er }}$ de Souabe. ${ }^{70}$ En ce qui concerne son épiscopat, on lui doit la réorganisation de la liturgie de l'Église salernitaine, la réfection du pavement du dôme et la construction de l'ambon. ${ }^{71}$

Son successeur fut Nicolas d'Aiello (11821221), qui régit l'épiscopat durant le difficile moment de transition du Royaume de Sicile des Normands aux Souabes. Il appartenait à une famille d'origine normande particuliè-

67 Cf. : Delogu - Peduto 2004.

68 CRISCI 1976. 213-223, 225-230, 231-238.

69 Galdi 2013. 100-105.

70 Pour une synthèse de son activité, cf. : OLdoni 2003. 400-403. Sur Chronicon cf. les essais introductifs de Giancarlo Andenna, Houbert Houben et Massimo Oldoni dans Bonetti 2001. 7-55.

71 CRISCI 1976. 239-260; GALDI 2000. 95-134. 
rement liée d'abord aux ducs ensuite aux rois normands de Sicile, dont le représentant le plus célèbre fut Mathieu ( $† 1193)$, père de Nicolas, un personnage central dans les évènements méridionaux de la deuxième moitié du XII ${ }^{\mathrm{e}}$ siècle et en particulier durant les règnes de Guillaume II et Tancrède d'Altavilla, auprès desquels il recouvrit les rôles de magister notarius, vicechancelier et chancelier. ${ }^{72}$ Mais Mathieu ne négligea pas Salerne, il commissiona des travaux dans la cathédrale et dota l'étabissement d'un hôpital pour les pauvres et les malades près de l'église de San Giovanni de Busandola, obtenue par son fils Nicolas en échange de celle de Santa Maria, de sa propriété, dans l'ancien quartier des Amalfitains. La fidélité de la famille d'Aiello à la maison normande fut partagée par Nicolas qui, en 1194, fut invité dans les territoires germaniques, et incarcéré pour sa position pro-normande - tellement favorable à l'élection de Tancrède d'Altavilla qu'il ordonna une conjuration contre Henri VI - et hostile aux Souabes. Malgré ces erreurs - ainsi que sa déclaration en faveur d'Otton IV de Brunswick - le nouveau roi de Sicile, l'empereur souabe Frédéric II ne le priva pas d'importantes marques de reconnaissance, comme celui de justicier in tota terra et hominubus Salernitane Ecclesie, en plus de confirmer à l'Église salernitaine, en 1221, ses droits et ses revenus, bien que le document qui le prouve nous soit parvenu uniquement en copie authentique de $1287 .^{73}$

Les années suivant la mort de Nicolas, comme on le sait, furent marquées par une détérioration des rapports entre Frédéric II et la Papauté, avec des conséquences prévisibles sur le plan diocésain ; bien que le gouvernement de Frédéric II ait été inauguré avec une intervention décisive d'Innocent III sur les modalités de l'élection épiscopale dans le sud

\footnotetext{
72 Sur les sources et l'historiographie de la famille d'Aiello et en particulier sur Mathieu, cf. : GALDI 2013. 90-100.

73 Sur l'épiscopat de Nicola, cf. : GALDI 2013. 94-96.
}

de l'Italie et un comportement plus que favorable de Frédéric par rapports aux dispositions papales. ${ }^{74}$ Les controverses entre l'empereur et le pape sur la nomination des évêques avaient déjà commencé durant le pontificat d'Honorius III, et le diocèse salernitain constitua justement un cas d'exemple, où c'est seulement en 1225 que le successeur de Nicolas, Cesario d'Alagno (1225-1263) a pu s'établir, après une nomination contestée non seulement par Frédéric II, mais par le chapitre même de la cathédrale. ${ }^{75}$ Les rapports entre Cesario - originaire d'Amalfi, où il avait d'abord été archidiacre de la cathédrale, puis évêque de Famagouste à partir de 1213 - et l'empereur, ne furent pas sans contrastes, même dans les années suivantes, comme le montrent les discordes relatives à l'élection de l'abbesse du monastère féminin de Saint Georges de Salerne. ${ }^{76}$

L'aggravation des dissensions entre la Papauté et les Souabes conditionnèrent aussi la succession de Cesario, et en effet la consécration du salernitain Mathieu della Porta (12631273) fut renvoyée en raison de l'opposition de Manfred, bien que son élection - survenue canoniquement de la part du chapitre - ait été confirmée par Urbain IV. ${ }^{77}$ Mais Salerne et l'Italie du sud devaient désormais s'ouvrir à une nouvelle dynastie : celle des Angevins. La fidélité de l'archevêque salernitain à la maison française - conséquence évidente aussi des rapports entre Mathieu et la Papauté ${ }^{78}$ - sont exemplairement démontrés par le fait que le premier acte émanant de Charles $\mathrm{I}^{\mathrm{er}}$ regardant Salerne est relatif à l'ordre adressé au justicier de la Principauté et terres de Bénévent de pourvoir à la restitution de certains biens de l'église de Santa Maria de Battipalla, usurpés par Galvano Lancia, sur lesquels Mathieu della

\footnotetext{
74 Cf. avec les références documentaires : CARUCCI 1943b. 16-20.

75 Carlo Carucci 1943b. 20-21.

76 Carlo Carucci 1943b. 21. Sur l'épiscopat de Cesario, cf. : CRISCI 1976. 282-291.

77 Carlo Carucci 1943b. 22-23.

78 À propos cf. : CRISCI 1976. 295-297.
} 
Porta dilectus consiliarius et familiaris du roi avait juridiction. ${ }^{79}$

Les années suivant la mort de Mathieu sont marquées par une période de vacance de l'épiscopat, bien qu'ait été supposée - sans preuves suffisantes - l'existence d'un archevêque du nom de Jean. ${ }^{80}$ L'élection de Philippe (1286-1298) se présente particulièrement tourmentée par des divisions internes au chapitre jusqu'à sa nomination par Honorius IV. Philippe fut l'une des personnalités les plus turbulentes de l'époque, suspecté même - dans les difficiles années de la guerre du Vespro - de sympathie pour les Aragonais de Sicile. Ses rapports avec Olevano, ancien fief du diocèse salernitain, furent difficiles ; de même avec le stratigotus, avec un médecin et familier du roi, avec certains chanoines, avec quelques représentants de familles salernitaines qui allèrent jusqu'à l'insulter publiquement dans la cathédrale au cours de la fête de saint Mathieu. Et, il entra en conflit avec les frères mineurs du couvent salernitain de saint Nicolas della Palma pour une question de ius funerandi et sepulturae. Controverses qui regardaient souvent la défense des intérêts patrimoniaux et juridictionnels de l'Église salernitaine, et qui s'inséraient dans une période marquée par de grands affrontements entre les classes sociales citadines, qui se poursuivront aussi au cours du siècle suivant et en y impliquant les évêques de la ville. ${ }^{81}$

- A.G. -

9 Cf. : GALDI 2011. 245.

${ }^{80}$ CRISCI 1976. 303-304.

${ }^{81}$ Du difficile épiscopat de Filippo, ainsi que des situations conflictuelles diffuses qui marquèrent la fin du XIII ${ }^{\mathrm{e}}$ siècle et une bonne partie du suivant, nous sommes bien informés par les Registres de la chancellerie angevine, cf. : GALDI 2011, en particulier 252-253 pour ce qui concerne Filippo.

\section{Organisation de l'espace et monumentalité}

\section{Potentiels d'une recherche liée au patrimoine}

Une définition préliminaire du contexte historique, en vue d'une minutieuse étude prosopographique des évêques et archevêques des diocèses européens, représente l'arrière-plan d'un intérêt concernant le potentiel du vaste champ de l'héritage culturel du clergé entre les $\mathrm{XII}^{\mathrm{e}}$ et $\mathrm{XV}^{\mathrm{e}}$ siècles. En particulier, le matériel épigraphique et monumental, fortement lié aux individus, dévoile explicitement les signes, les espaces et la propagande au sein de lieux de mémoire fondamentaux que les églises cathédrales représentent. ${ }^{82}$ Dans cette perspective, l'archidiocèse de Salerne représente un nombre conséquent de possibilités d'applications, car de nombreux approfondissements sur des questions liées à la localisation topographique de la cathédrale à l'intérieur de la ville de Salerne, à sa conception architecturale et à son évolution constructive jusqu'à l'analyse des aspects artistiques, sigillographiques et épigraphiques et funéraires, sont possibles. L'analyse prosopographique s'impose toutefois avec des caractéristiques d'interdisciplinarité entre la documentation écrite et matérielle qui caractérisera les futurs développements de la recherche.

Sur la base de ces prémisses, le travail a commencé à fournir des résultats à partir d'une sélection d'archevêques. L'image de Romualdo II Guarna de Salerne, par exemple, archevêque de 1153 à 1181, a démontré le potentiel de l'information directement liée à l'individu, à son action politique et sociale contenue non seulement dans les documents d'archives, mais aussi dans la cathédrale de Salerne, laquelle représente, bien évidement,

${ }^{82}$ Le projet GDRE a prévu un axe de recherche sur le recensement des objets artistiques, archéologiques et monumentaux. Cf. la présentation du projet dans ce même volume (n. du rédacteur- $\mathrm{G}$. Kiss). 
l'espace, l'écrin, réorganisé au fil du temps, et qui abrite les éléments sacrés, artistiques d'une promotion politique et sociale.

Dans la riche collection du musée diocésain "saint Mathieu » de Salerne est présent un sceau qui montre au recto : +R SAĹR II - ARCHIEPISĆO; au verso : SANCTVS : MATHEVS dans le champ saint Mathieu entre les lettres $\mathrm{S}$ et $\mathrm{M}$ abrégées (fig. 1a et 1b). L'objet, retrouvé en 1950, dans une urne avec des reliques, est l'unique sceau se rapportant aux archevêques de la période médiévale. ${ }^{83}$ Bien que certains chercheurs prétendent que pour la première fois la figure de saint Mathieu ait été choisie comme symbole de l'Église de Salerne, pour devenir ensuite le symbole de toute la ville, ${ }^{84}$ il est possible de vérifier, après une comparaison

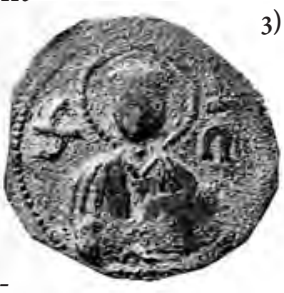
2 rapide avec des monnaies de l'hôtel de frappe d'époque normande de Salerne, objets très similaires, que le saint a déjà été représenté sur des émissions datées d'entre 1085 et 1111, au nom de Roger Borsa (fig. 2). N'étant présent dans la cathédrale aucun tombeau monumental explicitement attribuable à Romualdo II, il est même envisageable que les reliques contenues avec le sceau dans l'urne de 1950, soient les restes de l'archevêque Romualdo II. II était aussi un mécène de la cathédrale offrant l'ambon mineur, toujours préservé et en parfait état, qui présente dans la partie supérieure le nom de l'archevêque en mosaïque (fig. 3).

\section{L'espace physique, sacré, et politique de la cathédrale}

Aucune chronique ou document ne décrit les étapes de la construction de la nouvelle église cathédrale de la fin du XI ${ }^{\mathrm{e}}$ siècle. ${ }^{85}$ Un examen

\footnotetext{
83 Voir : D’Elia 2011. 40.

84 Voir : BracA 1997b. 13.

85 Avant la fin du $\mathrm{XI}^{\mathrm{e}}$ siècle, il y avait un autre édifice dont on n'a pas d'informations précises.
}
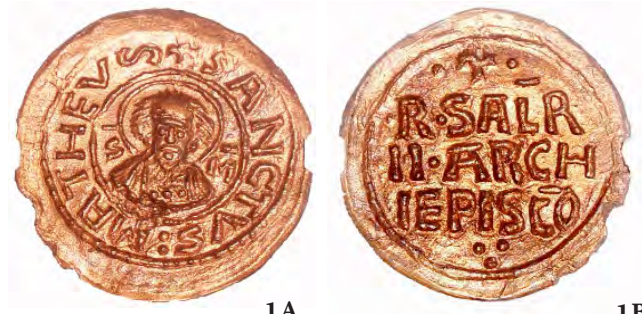

1) Sceau de Romualdo II Guarna conservé au Museo Dioceano "S. Matteo" di Salerno : a) Recto; $b$ ) Verso.

2) Monnaie de Roger Borsa (1085-1111)

3) Ambon "Guarna" de la cathédrale de Salerne comparé des fragments des sources historiques, littéraires et matérielles est indispensable pour clarifier la chronologie des travaux dont les chercheurs ont longtemps débattu. De cette façon, on peux affiner l'hypothèse selon laquelle la cathédrale serait la simple expression d'un désir de pacification avec les citoyens. ${ }^{86}$ La conquête de la ville lombarde par le normand Robert Guiscard, en 1076, est interprétée comme facteur impliquant la construction de la cathédrale. Pourtant, une lettre du pape Grégoire VII du 18 septembre 1080 tend à attribuer l'idée du nouveau projet de la cathédrale à d'autres raisons. Dans sa lettre, adressée à l'archevêque lombard Alfano I ${ }^{\text {er }}$, le pape, en entendant les nouvelles de la redécouverte des

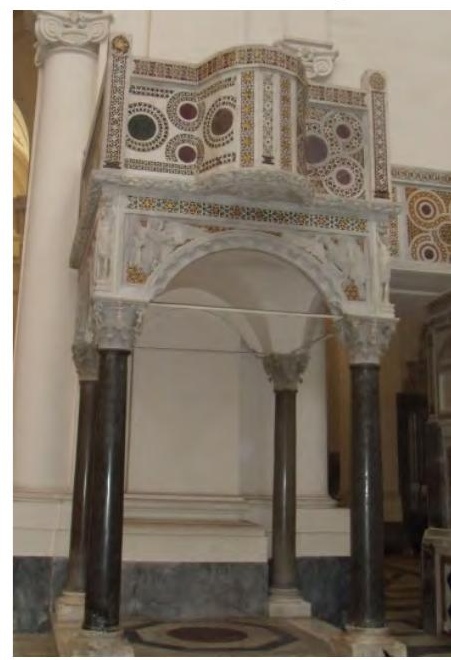
restes de saint Mathieu, suggère à l'archevêque de solliciter « il glorioso duca Roberto e la sua nobilissima Consorte, a mostrarsi degni di un

86 Voir : Arturo CARUCCI 1986, 24 et nr. 3, 51. Sur la relation entre les citoyens et la nouvelle cathédrale, cf. aussi: SANGERMANO 2000. 53-76. 


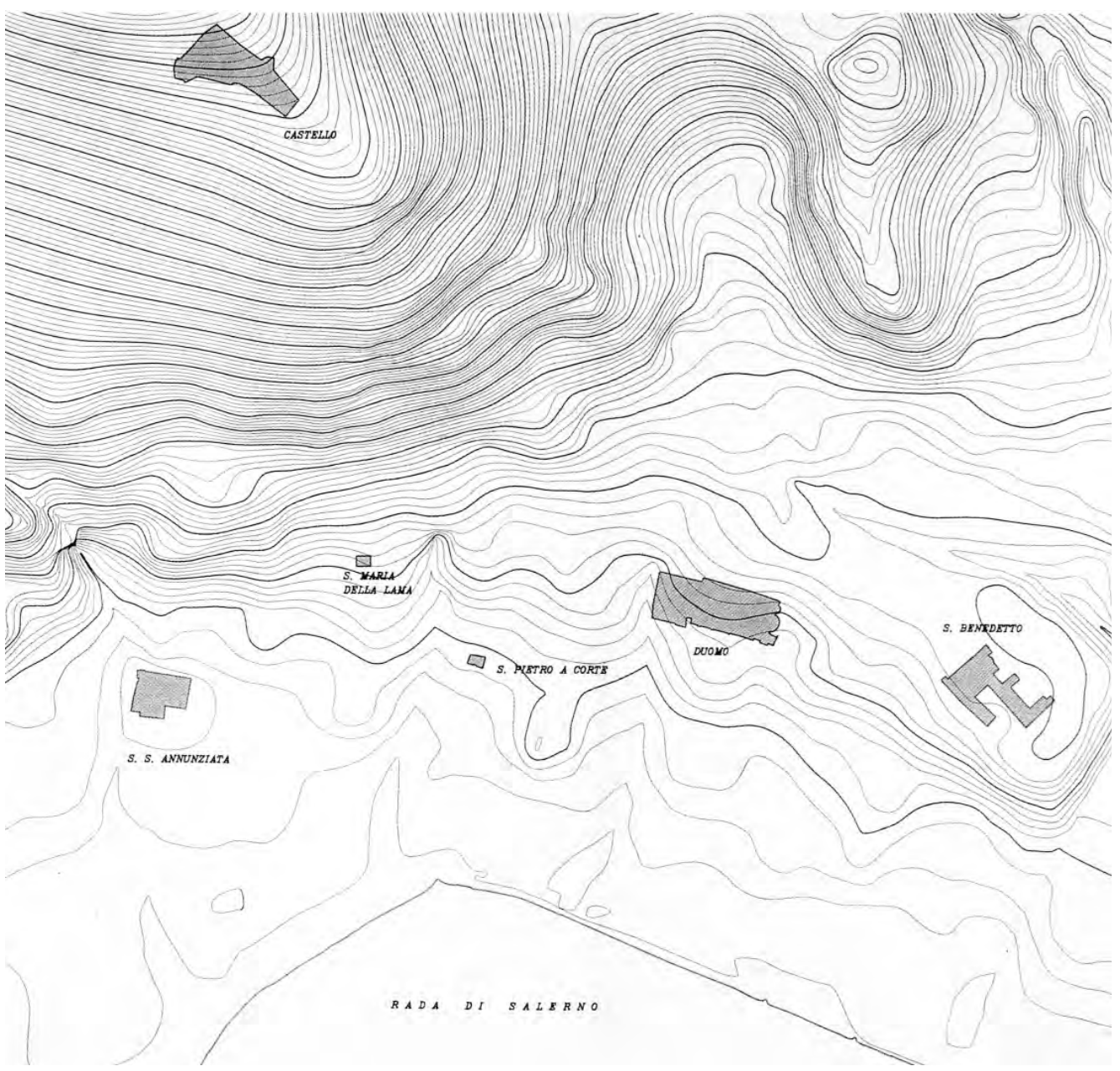

4) Monuments et morphologie du terrain du centre de Salerne (CIFELLI - SANTORO 1995)

così insigne Patrono che a loro si è compiaciuto rivelarsi; a lui portino riverenza e devozione e, con l'ossequio profondo, cerchino di assicurarsi per sé e i loro sudditi la grazia e la protezione». ${ }^{87}$ L'importance des reliques du saint évangéliste nécessite une collocation adéquate, laquelle n'a été vraisemblablement ni l'ancienne cathédrale, ni la nouvelle crypte récemment édifiée pour accueillir les nombreuses reliques retrouvées justement par l'archevêque Alfano,

87 La lettre de Grégoire VII, datée du 18 septembre 1080 est mentionnée par Marsilio CoLonNa 1580.69-70., aussi dans : CAPONE 1927-1929. I. 11-12. après l'arrivée de Guiscard à Salerne. Par conséquent, l'archevêque lombard Alfano, en accord avec le duc normand, commence la grandiose construction dont l'archétype est érigée au Mont Cassin quelques années auparavant (1066-1071). Les caractéristiques architecturales de l'église du Latium sont reprises et perfectionnées pour donner à Salerne une nouvelle cathédrale digne de son opulence et du célèbre saint Mathieu. ${ }^{88}$ La cathédrale

88 Aucun attestation précise n'assure qu'Alfano a réalisé le projet. Il a une vaste culture et il est poète, érudit et médecin, mais il n’est jamais appelé architecte 


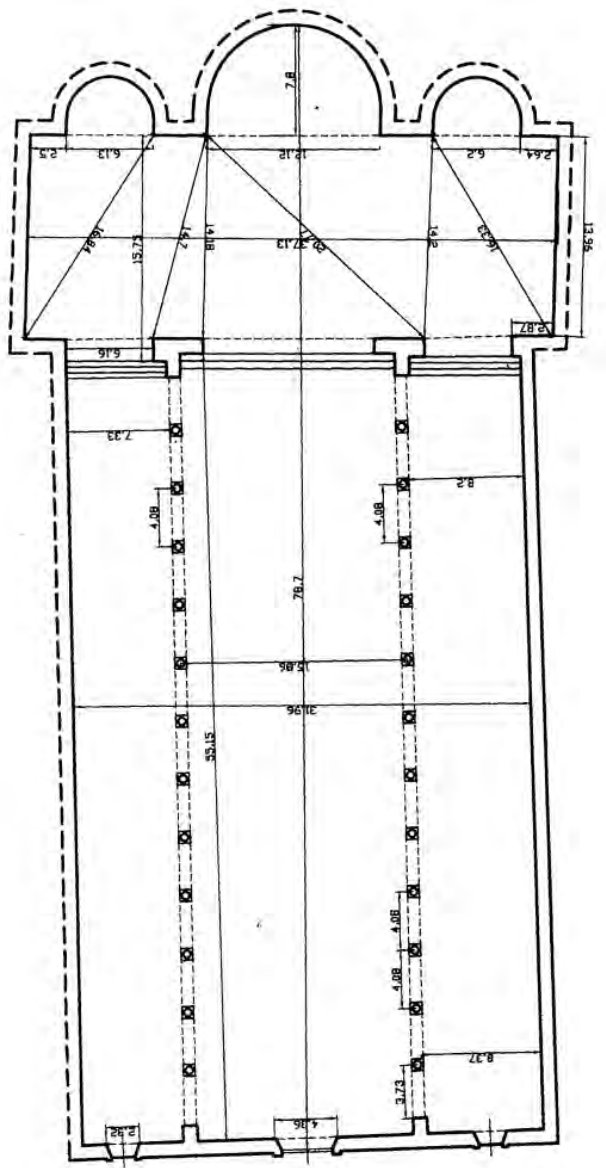

5) Plan de la cathédrale de Salerne entre la fin $d u X^{e}$ et le début du XII ${ }^{e}$ siècle (BRACA, 2011)

a été achevée pour l'essentiel en 1085, l'année même où elle est vraisemblablement consacrée par le pape Grégoire VII, en exil à Salerne. ${ }^{89} \mathrm{Il}$ manque cependant les œuvres d'art qui en ont fait, au fil du temps, un sanctuaire de référence pour de nombreux fidèles : la porte en bronze,

ou magister. Les choix typologiques auraient été attribués à Alfano. L'église semble être le résultat d'une élaboration idéologique et technique d'Alfano, connaissances vraisemblablement apportées par Robert Guiscard, qui en finance la construction. L'église est un nouvel élément dans l'architecture de l'Italie normande.

89 De nombreuses citations rappellent la consécration par le pape. Cf. : CAPONE 1927-1929. I. 39-41. les ambons, les sols en mosaïque du transept et du chœur, les parapets, le clocher, les tombeaux seigneuriaux, en particulier de la reine Marguerite de Durazzo.

L'espace choisi pour la construction de la cathédrale est le résultat de certains choix obligés. Le premier élément à considérer est l'état de l'urbanisme. Le nouvel édifice est situé dans un emplacement périphérique du centre de la ville médiévale de Salerne, en raison notamment de ses imposantes dimensions qui ne peuvent pas être insérées dans les zones habitées proches de la mer, déjà occupées par d'autres importants bâtiments plus anciens. Ce choix est dû à la nouvelle planification et à la croissance urbaine à l'époque normande qui s'effectue dans le quartier Orto Magno, jusquelà occupé par de nombreux terrains cultivés et potagers. Ici émergent les bâtiments normands les plus importants ; à côté du monastère de Saint-Benoît, Robert Guiscard érige sa résidence, connue sous le nom Castel Terracena et, à quelques mètres de distance, est également construit le palais de l'archevêque, très proche de la cathédrale.

Parmi les éléments déterminant le choix du site, il y a aussi le conditionnement morphologique de la ville qui se développe de la mer jusqu'au mont Bonadies. La zone, en effet, est la seule en position élevée à présenter un ample espace naturellement prêt à être nivelé, bien que non idéal. Le terrain, en ce point, est le résultat d'anciens éboulements et se trouve au milieu de deux dépressions où coulent deux torrents (fig. 4). ${ }^{90} \mathrm{Il}$ ne reste donc qu'à opter pour une zone située entre les espaces libres, la construction et les ajouts ultérieurs étant influencés par la morphologie : si l'on observe le plan de l'église normande on constate immédiatement une anomalie dans la construction non-linéaire des nefs ; la nef au sud est plus courte que les autres, ce qui provoque des modifications conséquentes des caractères architecturaux de la construction

90 Cf. : Cifelli - Santoro 1995 ; Miccio 2011. 337. 


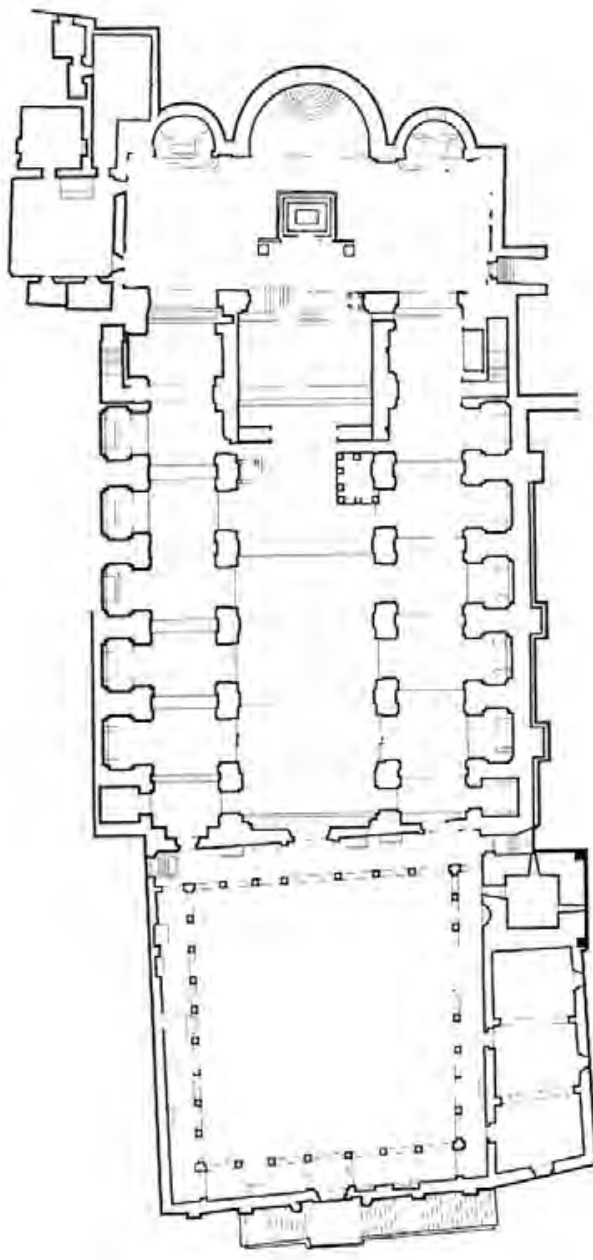

6) Plan de la cathédrale complétée au XII siècle et restaurée à l'époque moderne

(fig. 5). ${ }^{91}$ Les ajouts à la construction initiale doivent donc tenir compte de certains problèmes statiques et d'équilibre global. Même la construction du portique au $\mathrm{XII}^{\mathrm{e}}$ siècle est en fait déterminée par la nécessité de trouver une solution qui rétablisse une solidité architecturale au monument. Sur l'ensemble du côté sud, un profond dénivelé est encore évident, indiquant un niveau du sol plus bas par rapport au côté nord. Une référence chronologique

${ }^{91}$ Leétude globale la plus récente sur la cathédrale a été realisée par Antonio Braca (BRACA 2013). nous est également donnée par l'épigraphe placée sur la porte d'entrée du portique (la porte des lions), vu que la datation ne dépasse pas la troisième décennie du XII ${ }^{\mathrm{e}}$ siècle. Même le clocher, du milieu du XII ${ }^{\mathrm{e}}$ siècle, est construit dans la partie sud du bâtiment afin de soutenir l'édifice (fig. 6).

\section{L'espace interne de la cathédrale}

Les motivations d'un raisonnement sur certains éléments situés à l'intérieur de la cathédrale, en particulier les tombes monumentales, sont dues à la nécessité historique qui implique et tente d'identifier une signification symbolique pour les hommes du Moyen Age. Le tombeau est à la fois représentation artistique, épigraphique, sociale et politique.

Les tombeaux de la cathédrale de Salerne font l'objet d'une analyse qui n'a jamais été véritablement développée, qui pourra être un outil important pour atteindre des résultats en termes de prosopographie. L'analyse comprendrait:a) la collocation originale et les positions secondaires occupées par les monuments funéraires au fil du temps, à travers l'information dans les dessins et manuscrits. Souvent, il était nécessaire de déplacer les tombeaux, par conséquent la reconstitution topographique implique également des problèmes d'attribution et d'interprétation ; b) l'analyse épigraphique. Actuellement effacé ou endommagé, le matériel épigraphique a été transcrit dans certains manuscrits de 1700 et 1800 , et est à réexaminer pour une contextualisation appropriée ; c) l'analyse des blasons et des armoiries. Concrètement jamais réalisée, cette analyse pourrait changer certaines attributions ; d) le réemploi des anciens sarcophages d'époque classique permet aisément de supposer l'approvisionnement, le transport et le commerce, ainsi que des critères de choix.

L'étude s'est focalisée sur les évêques et les archevêques, mais peut être étendue aux personnages importants comme des nobles, les bourgeois, ou les souverains. On présente 


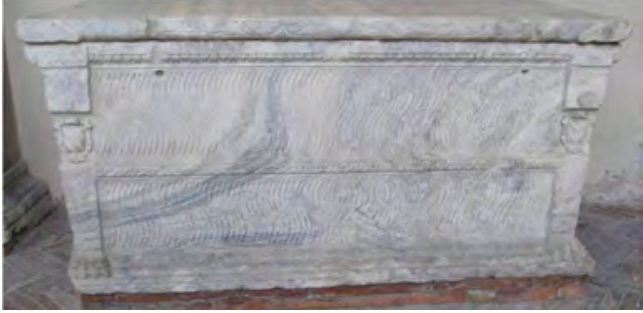

7) Sarcophage « Guarna»

ici le cas du supposé sarcophage Guarna, aujourd'hui présent dans le portique de la cathédrale, pour souligner comment certaines interprétations historiographiques contradictoires peuvent compliquer plutôt que clarifier les attributions (fig. 7).

La tradition locale veut que le sarcophage strigilé, désormais en position secondaire, placé près de l'entrée de l'église, contenait les restes de l'évêque Romuald II Guarna. Le sarcophage, daté d'entre les $\mathrm{III}^{\mathrm{e}}$ et $\mathrm{IV}^{\mathrm{e}}$ siècles, ne présente pas d'épigraphe ni de détails sculpturaux très élaborés. Le couvercle, très simple, est compatible avec les dimensions et le marbre du sarcophage qui n'a pas été remplacé au fil des ans. Sur les côtés, se présentent deux blasons ayant été ajoutés à l'époque moderne, lesquels sont similaires à ceux de la famille Santomango. Pourquoi alors attribuer le monument à la famille Guarna et à l'archevêque Romuald II ? En 1921, Alfredo De Crescenzo a écrit un commentaire sur deux documents de la seconde moitié du $\mathrm{XVI}^{\mathrm{e}}$ siècle, expliquant l'attribution de ce sarcophage à la famille Guarna. Il est en effet convaincu que les représentations héraldiques présentes sur les petits piliers sont de la famille Guarna et non des Santomango (fig. 8). ${ }^{92}$ En outre, Michele De Angelis confirma en 1933 reconnaître les symboles de la famille Guarna en les confrontant avec les emblèmes présents, déjà avant 1763, dans la Salle des Armes du palais de l'archevêque de Salerne. ${ }^{93}$ Selon les deux chercheurs, la confusion pourrait être due à un changement de l'image

\footnotetext{
92 Cf. : De Crescenzo 1921.

93 Cf. : De ANGelis 1933.
}

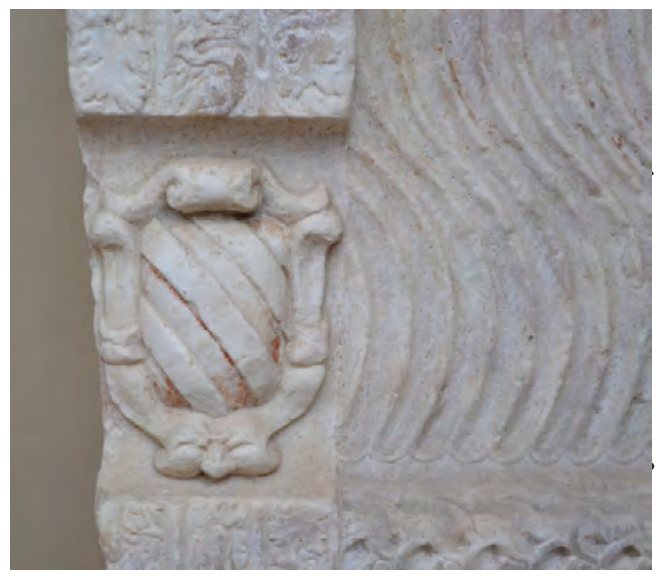

8) Détail du sarcophage «Guarna »

Guarna qui aurait changé complètement au cours de l'époque moderne, en particulier pour devenir identiques à ceux d'une autre famille (Santomango). De plus, le manuscrit Pinto, source historique du XVIII ${ }^{\mathrm{e}}$ siècle, particulièrement précis quant aux origines des familles nobles salernitaines, et plus spécifiquement attentif à la reproduction des symboles héraldiques (fig. 9), ainsi qu'aux œuvres relatives aux personnages importants et leurs monuments, ne cite aucune sépulture ou tombeau de Romuald II, (alors qu'il mentionne le tombeau d'autres archevêques) et propose l'emblème des Santomango tel qu'il est représenté sur le sarcophage en question dans le portique. ${ }^{95} \mathrm{Il}$ est donc évident qu'il y a beaucoup à apprendre des tombeaux qui ne portent pas d'indication épigraphiques explicites, en appliquant une méthodologie plus rigoureuse et une étude sur les emplacements qui se sont succédés à l'intérieur de la cathédrale. ${ }^{96}$

94 En réalité, les deux chercheurs, sans avancer d'arguments particuliers, le croient être enterré dans le même sarcophage que l'archevêque Romualdo $\mathrm{I}^{\mathrm{er}}$.

95 Cf. : GALdi 2013., en particulier 100-105.

96 Récemment, certaines précautions méthodologiques ont été remises en question dans : HERKLOTz 2001; GARMS - JUfFINGER - WARD PERKINS 1981 ; GARMS SOMMERleChNER - TelesKo 1994 ; GARMS - RomANINI 1990. 


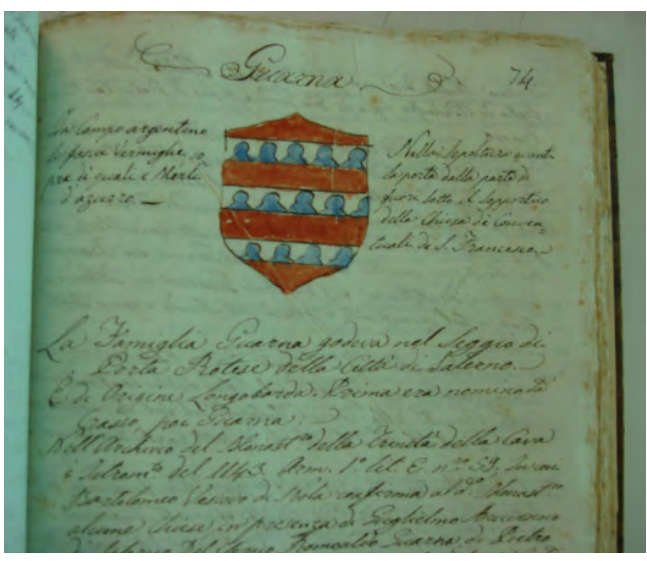

9) Armoiries de la famille Guarna dans le Manuscrit "Pinto» conservé à la Bibliothèque Provinciale de Salerne

Dans la cathédrale de Salerne, le principal modèle de tombeau de la fin du $\mathrm{XI}^{\mathrm{e}}$ siècle que l'on trouve, est sans aucun doute le sarcophage du pape Hildebrand de Soana (Grégoire VII), grâce auquel on pourrait expliquer le réemploi d'une grande quantité de sarcophages d'époque romaine (fig. 10). ${ }^{97}$ Dans le contexte ecclésiastique de la fin des $\mathrm{XI}^{\mathrm{e}}-\mathrm{XII}^{\mathrm{e}}$ siècles, naît la nécessité de réutiliser, pour les défunts illustres, d'anciens sarcophages romains conservant les dessins originaux avec seuls quelques petits changements. À partir du XIII ${ }^{\mathrm{e}}$ siècle, laïques et bourgeois commencent également à réemployer des sarcophages d'époque romaine mais souvent avec des modifications plus importantes : couvercle, signes épigraphiques, ajouts de l'héraldique, transformations sculpturales.

La cathédrale de saint Mathieu devient avec le temps une église-sanctuaire dans laquelle, qui se recueillait sur les tombes du saint et du pape Grégoire VII, avait l'occasion d'admirer également des sarcophages, des gisants, des inscriptions et des armoiries de personnages illustres laïcs et ecclésiastiques. La présence de monuments funéraires regardant des personnalités importantes issues aussi de

${ }_{97}$ Sur la tombe du pape, cf. aussi : HerkLotz 2001. 144-146.

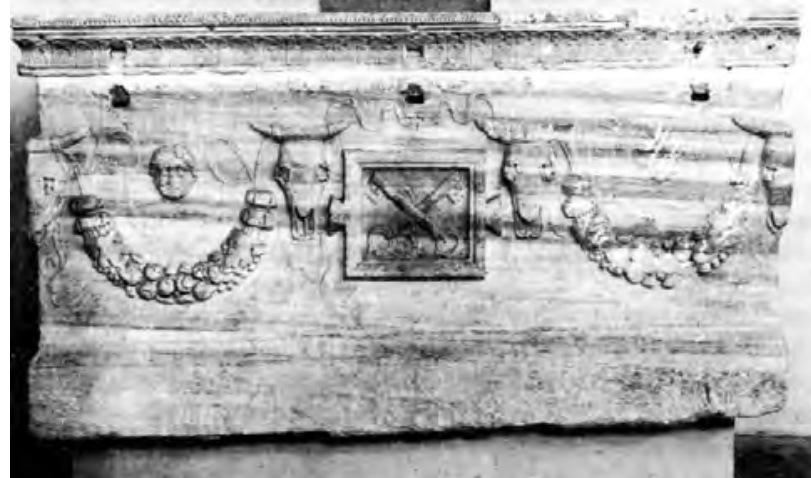

10) Sarcophage du pape Grégoire VII

la noblesse de la ville de Salerne advient au fil du temps au sein de l'espace l'ecclésiastique, en imitant le sarcophage du pape qui rappelle et célèbre le Saint-Siège à Rome. La référence à la romanité est du reste explicite dans l'épigraphe présente sur la façade de la cathédrale aux alentours de 1084, imposée par le duc Robert Guiscard et Alfano Ir ${ }^{\mathrm{er}} \mathrm{M} \cdot \mathrm{A} \cdot \mathrm{ET}$ EVANGELISTAE PATRONO VRBIS - ROBBERTVS DVX - R · IMP · MAXIM - TRIVMPHATOR - DE AERARIO PECULIARI. ${ }^{98}$

Les sarcophages de la cathédrale sont comparables à d'autres sarcophages réemployés dans les églises de Naples, Amalfi, Capoue, Cava de' Tirreni bien qu'à Salerne ils soient plus nombreux (plus de trente). Ces chiffres suggèrent l'existence d'un marché d'objets récupérés sur des sites romains de grande importance. Paestum, Stabia et Pouzzoles semblent être les sources d'approvisionnement les plus probables, car à partir de ces sites, le transport maritime permet de

98 Une traduction: « Le duc Robert, grand vainqueur de l'empire romain, à ses propres frais (offre) à Mathieu le saint patron, apôtre et évangéliste de la ville. » L'étude de cette épigraphe mérite également un approfondissement philologique et archéologique. Jusqu'à présent, beaucoup de chercheurs ont mentionné tout simplement le texte, mais je voudrais signaler les explications récentes de Giovanna Assunta Lanzetta qui a produit une étude préliminaire, malheureusement inédite. 


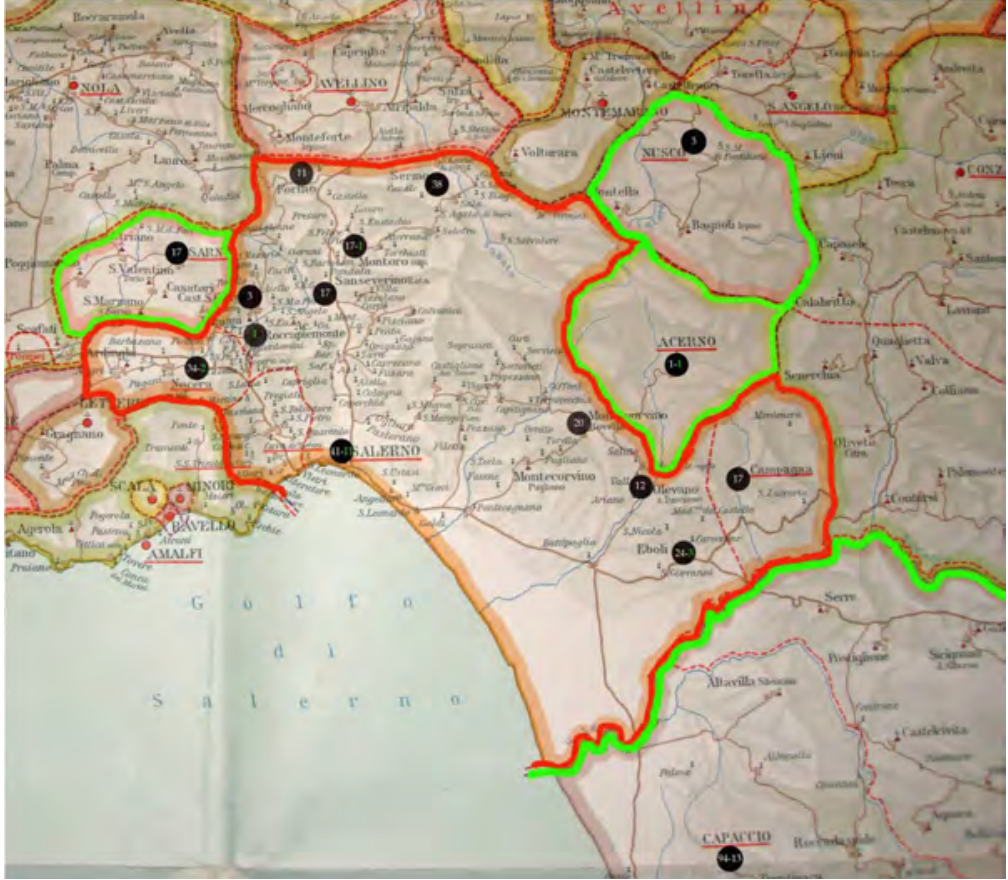

11) Carte des églises et des monastères cités dans les Rationes Decimarum Italie (Campaniae) : Diocèse de Salerne

simplifier les déplacements qui restent plus difficiles (mais pas impossibles) qu'à partir de sites intérieurs tels que Nocera, Bénévent, Santa Maria Capua Vetere.

Rome même se situe parmi les lieux d'approvisionnement d'origine de certains sarcophages parce que Robert Guiscard réclamé par le pape Grégoire VII, assiégé dans Castel Sant'Angelo par les troupes de l'empereur Henri IV, a organisé une expédition en juin 1083. Le 21 mai 1084, Robert arrivé à Rome avec des milliers d'hommes, commençait à piller la ville, forçant ainsi l'empereur à se retirer. Au terme du sac, Robert accompagna le pape à Salerne pour le protéger d'une contre-attaque impériale, transportant vraisemblablement aussi quelques vestiges dont plusieurs sarcophages.
L'espace et les limites $d u$ diocèse de Salerne entre les $\mathrm{XII}^{e}-\mathrm{XV} \boldsymbol{V}^{e}$ siècles

Une analyse de l'espace géographique et administratif du diocèse, avec cartographie et classement des églises médiévales (ou de leurs restes) entre le $\mathrm{XII}^{\mathrm{e}}$ et le $\mathrm{XV}^{\mathrm{e}}$ siècle, est en cours. Une reconstitution en ce sens, pour les $\mathrm{XI}^{\mathrm{e}}-\mathrm{XII}^{\mathrm{e}}$ siècles, a déjà été proposée par Amalia Galdi dans une étude fondamentale de la Campanie médiévale. ${ }^{99}$

Une tentative de reconstitution pour les années 1308-1310, d'après les informations fournies par les Rationes Decimarum, est ici présentée afin d'obtenir une représentation détaillée de l'archidiocèse sur un laps de temps très court, ${ }^{100}$ même s'il subsiste de nombreuses ambiguïtés à propos de certains noms de lieux faisant référence à des églises appartenant à d'autres diocèses voisins. Ces doutes persistent également en raison du manque de clarté de la part des éditeurs des Rationes qui, dans l'introduction, ne fournissent pas d'éléments certains, rendant ainsi l'information peu fiable. La confusion demeure même pour d'autres régions telles que les Pouilles.

L'étude géographique ouvre des pistes de recherche sur des questions de juridiction et de la sphère économique, fiscale et patrimoniale qui représentera un stade ultérieur de recherches. Les relations entre les diocèses suffragants et le diocèse de Salerne, ainsi que

99 Cf. : GALdi 2004a, entre 96 et 97.

100 Iguanez - Mattei - Cerasoli - Sella 1942. 


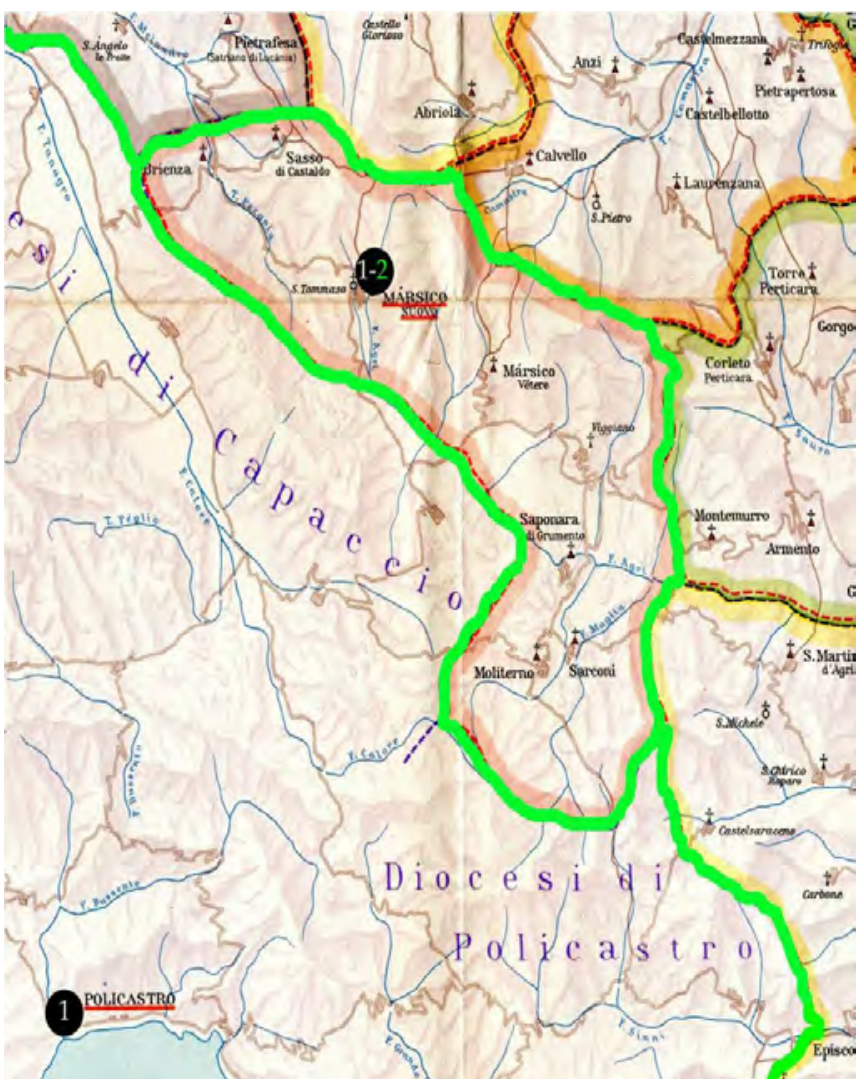

fait partie du territoire du diocèse de Salerne, probablement à partir de la fin du XIII ${ }^{\mathrm{e}}$ siècle, dans des circonstances historiques peu claires, pouvant représenter un développement ultérieur de la recherche en cours.

Parmi les diocèses suffragants Sarno, Capaccio, Acerno, Nusco, Marsico, Policastro, les deux premiers semblent les plus riches car ils payent les dîmes les plus élevées. Les raisons en devraient être liées à leur situation géographique. Sarno est un point de référence de la zone très fertile au pied du Vésuve, tandis que Capaccio est au milieu de la plaine riche et tout aussi florissante de la rivière Sele. Si les données quantitatives relatives aux églises sont explicites dans la reconstitution proposée, les confirmations quant à la qua-

12) Carte des églises et des monastères (élaboration à partir des Rationes Decimarum Italie, Campaniae) : Archidiocèse de Salerne (partie sud)

les rapports entre l'archidiocèse et les monastères, surtout de Cava de' Tirreni, qui conserve de nombreuses enclaves jusqu'à la fin de la guerre des Vêpres, dans la première moitié du $\mathrm{XIV}^{\mathrm{e}}$ siècle, méritent une attention particulière.

Pour le moment, l'analyse peut esquisser pour le début XIV ${ }^{\mathrm{e}}$ un territoire diocésain beaucoup plus restreint par rapport à la période de la fin $\mathrm{du} \mathrm{XI}^{\mathrm{e}}-\mathrm{XII}^{\mathrm{e}}$ siècle, mais elle met en évidence les territoires les plus riches, en citant les nombreuses églises de certaines dépendances diocésaines comme Montoro, Montecorvino, Olevano, Nocera (fig. 11-12). ${ }^{101}$ Cette dernière

101 Dans les cartes proposées les chiffres sur la gauche sont liés aux églises ; les chiffres à droite appartiennent aux monastères. Dans tous les cas où il y a un seul numéro, il est fait référence à des églises, sauf lité et à la valeur patrimoniale des églises individuelles pourront émerger d'une analyse comptable et économique des dîmes, qui n'en est qu'au début.

Si le potentiel pour les années 1308-1310 est assez évident et d'un approfondissement assez aisé, le travail est beaucoup plus complexe pour d'autres périodes historiques pour lesquelles il n'a été fait, jusqu'à présent, aucune démarche en vue d'étudier les frontières et la juridiction. Dans l'état actuel de la recherche, une sélection préliminaire parmi les documents décrivant le patrimoine et les limites du diocèse, conservés au Musée Diocésain de Salerne (dont de nombreux sont encore inédits) a été ébauchée. Une recherche à long terme serait porteuse de grandes opportunités tempérées cependant de complexité. *

- A. M. S. -

dans le cas de Roccapiemonte puisque le nombre se réfère au seul monastère. 


\section{ABRÉVIATIONS, BIBLIOGRAPHIE ${ }^{102}$}

Abignente, Giovanni (1888): Le chartulae fraternitatis ed il Libro de confratres della Chiesa salernitana. Archivio storico per le Province napoletane. XIII. nr. 1. 450-483.

Aceto, Francesco (2001): La sculpture à la court angevine. In: L'Europe des Anjous. Aventure des princes angevins $d u \mathrm{XIII}^{e}$ au XIV $\mathrm{XI}^{e}$ siècle. Paris, Somogy Art. 75-88.

ACOCELla, Nicola (1958): La figura el'opera di Alfano I di Salerno (sec. XI): profilo biografico. Rassegna Storica Salernitana. XIX. nr. 1-4. 1-74.

ACOCELla, NiCola (1959): La figura el'opera di Alfano I di Salerno (sec. XI): Alfano nella critica moderna. Rassegna Storica Salernitana. XX.nr. 1-4. 17-90.

Acocella, Nicola (1963-1964): Il canonico Giuseppe Paesano storico della Chiesa salernitana. Rassegna Storica Salernitana. 24-25. 139-156.

Amarotta, Arcangelo R. (1989): Salerno romana e medievale. Salerno, Pietro Laveglia.

Amatuccio, Giovanni (2012): Un nome per l'ignoto cavaliere della cattedrale di Salerno. Rassegna Storica Salernitana. N.s. 57. 81-96.

Amarotta, Arcangelo Raffaele - Iannelli, Maria Antonietta (1990): Medioevo sepolto a Salerno. S. Grammazio a li Canali. Atti dell'Accademia Pontaniana. N.s. 39. 5-46.

Andenna, Giancarlo - Picasso, Giorgio (ed.) (1996): Longobardia e Longobardi nell'Italia meridionale. Le istituzioni ecclesiastiche. Actes du IIe Colloque international d'études promus par le Centre culturel de l'Université Catholique du Sacré Coeur (Bénévent, 29-31 mai 1992). Milan, Università Cattolica.

Avagliano, Lucio (1972): Terre e feudi della Chiesa del Mezzogiorno. I beni della Mensa Arcivescovile di Salerno nei secoli XVI-XVII. Salerno, Libreria Internazionale.

Avallone, Ricardo (1968): Alfanus Salernitanus lumen Europae saeculo XI. Rivista di studi salernitani. I. nr. 1. (gennaio-giugno) 107-133.

Avallone, Ricardo (1997): Il manoscritto Pinto nella Biblioteca Provinciale di Salerno. Il Follaro. Rivista economica della Camera di Commercio di Salerno. N.s. XLI. nr. 3. 89-95.

Aventin, Laurence (2005): L'image au lieu de la liturgie. Le décor du pupitre de l'évangile de l'ambon d'Aiello dans la cathédrale de Salerne

102 Les références bibliographiques présentées dans les notes, se réfèrent seulement à quelques-uns des textes cités dans l'entière bibliographie pour des raisons d'espace. et ses principales variantes. Mélanges de l'Ecole Française de Rome. 117. nr. 1. 323-352.

BAlducci, Antonio (1945): L'Archivio della Curia arcivescovile di Salerno. Regesto delle pergamene (945-1727). Rassegna Storica Salernitana VI. nr. 3-4 (luglio-dicembre) 248-344.

Balducci, Antonio (1953): L'altare maggiore del Duomo diSalerno. Rassegna Storica Salernitana. XIV. nr. 3-4 (luglio-dicembre) 186-195.

Balducci, Antonio (1954): L'Archivio della Curia arcivescovile di Salerno. I registri della Mensa. Rassegna Storica Salernitana. XV. nr. 1-4. 63-150.

BALDUCCI, Antonio (1954a): La traslazione di S. Matteo a Salerno ed un'ipotesi del Garufi. Rassegna Storica Salernitana. XV. nr. 1-4. 47-50.

BALDUCCI, ANTONIo (1963-1964): Prima visita pastorale dell'arcivescovo Marsili Colonna a Salerno nel 1575. Rassegna Storica Salernitana.XXIV-XXV. 103-136.

BAlducci, Antonio (1957): Una lapide di Alfano I del 1078 e la data di inizio della costruzione del Duomo diSalerno. Rassegna Storica Salernitana.XVIII. nr. 1-4. 156-161.

Bergamo, Giuseppe (1973-1974): Costruzioni e ricostruzioni nell'archidiocesi di Salerno e nell'amministrazione perpetua di Acerno. I-V. Battipaglia, Graf sud.

Bock, Nicolas (2001): L'art à la court angevine : la sculpture et le gothique International. In: Aceto, Francesco (éd.): L'Europe des Anjou. Aventure des princes angevins $d u \mathrm{XIII}^{e}$ au XIV ${ }^{e}$ siècle. Paris, Somogy Edition d'art. 89-101.

Bock, Nicolas (2001a): Kunst am Hofe der AnjouDurazzo. Der Bildhauer Antonio Baboccio (1351 um 1423), München, Deutscher Verlag.

Bonetti, Cinzia (ed.) (2001): Romualdo Guarna, Chronicon. Cava dei Tirreni, Avagliano.

Braca, Antonio (1990): La cappella del tesoro del duomo di Salerno, Salerno, Laveglia-Carlone.

Braca, Antonio (1995): Sculture trecentesche nel duomo di Salerno. Rassegna Storica Salernitana. N.s. 24. 97-119.

Braca, Antonio (1997a): Oltre Montecassino, la pianta originaria del duomo diSalerno. Rassegna Storica Salernitana. N.s. 27. 7-42.

Braca, Antonio (1997b): Matthaee Pater. Immagini di San Matteo a Salerno. Salerno, Incisivo.

Braca, Antonio (1998a): Interventi nel Duomo di Salerno, dopo il terremoto del 1688. In: Cioffi, Maria Cristina (ed.): Il Barocco a Salerno. Salerno, Laveglia Editore. 65-90. 
Braca, Antonio (1998b): Le lastre a mosaico dal duomo di Salerno. Rassegna Storica Salernitana. N.s. 30. 7-42.

Braca, Antonio (2001): Salerno e la forma urbis: iconografia ragionata della città dal Medioevo all'età moderna. In: Musi, Aurelio - Oldoni, Massimo Placanica, Augusto (eds): Storia dell'Università di Salerno. Salerno, Arti Grafiche Boccia. 357-395.

Braca, Antonio (2002): La schola cantorum e gli amboni del duomo di Salerno. Schola Salernitana. Annali. V-VI. 111-156.

Braca, Antonio (2003): Il Duomo di Salerno. Architettura e opere d'arte del Medioevo e dell'Età moderna. Nocera Inferiore, Laveglia.

BracA, Antonio (2004): I mosaici del transetto e del coro del duomo di Salerno. In: DELOGU - PEDUTO 2004. 238-277.

Braca, Antonio (2011): La cattedrale di San Matteo. In: Campanelli, Adele (ed.): Dopo lo tsunami Salerno antica. Napoli, Arte'm. 324-335.

Caiazza Pietro (1975): Aspetti e problemi di Alfano I arcivescovo salernitano: Benedictina. XXII. fasc. $1-2.347-358$.

Capone, Arturo (1927-1929): Il duomo di Salerno. I-II. Salerno, Di Giacomo.

CApone, Arturo (1943): Il « ius scannagii seu cultelli » del Capitolo della Cattedrale di Salerno. Rassegna Storica Salernitana.IV.nr. 1-2 (gennaio-giugno). 33-40.

CAPuto, Ottavio (1976): I vescovi nati nelle diocesi di Salerno e Acerno, Salerno, Edizioni della Curia Arcivescovile.

Carucci, Arturo (1951): L' "Exultet » di Salerno. Miniature del secolo XIII. Salerno, [s.a.] Tipografia Jannone.

CARUCCI, ARturo (1974): Lelapidi di Alfano I in Salerno. Benedictina 21.29-52.

Carucci, Arturo (1978): Il duomo di Salerno e il suo Museo. Salerno, Scuola Tipografica Istituto Anselmi.

Carucci, Arturo (1986): La cattedrale di Salerno. (Marigliano [NA], Istituto Anselmi].

Carucci, Arturo (1998): Il Chronicon Salernitanum (sec. X), Salerno, Salernum.

Carucci, Arturo (1990): IX centenario di una leggenda affrescata nel Duomo di Salerno. Bollettino storico di Salerno e Principato Citra. VIII. nr. 1. 25-34.

CARUCCI, Arturo (2002): Alfano nell'arte e nella storia. Il Picentino. Organo della Società economica della Provincia di Salerno. XLVII. (gennaiogiugno) 15-31.

Carucci, Carlo (1943a): La Chiesa maggiore di Salerno nella politica ecclesiastica della casa sveva. Archivio storico per la provincia di Salerno. N.s. I. (gennaio-dicembre) 323-347.

Carucci, Carlo (1943b): Una svolta nelle elezioni episcopali vista nella Diocesi di Salerno. Rassegna Storica Salernitana. IV.nr. 1-2 (gennaio-giugno) $16-26$.

CASPAR, ERICH (ed.) (1923): Das Register Gregors VII. Teil 2. Hannover, Weidmannsche Buchhandlung.

Cassese, Leopoldo (1938): La Platea generale della Chiesa salernitana del sec. XVIII. Rassegna Storica Salernitana. II. nr. 2. (agosto). 307-322.

Chierici, Gino (1937): Il duomo di Salerno e la chiesa di Montecassino, in Rassegna Storica Salernitana. I. nr. 1. (giugno) 95-109.

Cifelli, Ferdinando - Santoro, Ugo (1995): Il centro antico di Salerno attraverso l'analisi dell'ambiente fisico. Apollo. Bollettino dei Musei Provinciali del Salernitano. Nr. XI. 102-115.

Cilento, Nicola (1969): La Chiesa di Napoli nell'Alto Medioevo. In: Storia di Napoli. II:2. 1969. Napoli, Società editrice Storia di Napoli. 641-735.

Crisci, Generoso (1976): Il cammino della Chiesa salernitana nell'opera dei suoi vescovi (sec. V$X X I)$. I. Napoli-Roma. Libreria Editrice Redenzione.

Crisci, Generoso (2001): Salerno Sacra. Ricerche storiche. I-II-III. II edizione riveduta e integrata a cura di Vincenzo de Simone, Giuseppe Rescigno, Francesco Manzione, Donato De Mattia. Lancusi, Gutemberg.

Cuozzo, Errico (1975): Un vescovo della Longobardia minore. Alfano arcivescovo di Salerno. Campania Sacra. 6. 15-29.

D’Addosio, Giuseppe B. (1909): Illustrazioni e documenti sulle carte di S. Andrea in Amalfi e S. Matteo in Salerno. Archivio storico per le Province napoletane. XXXIV. nr. 1. 19-48.

D'Ajello, Gaetano (1989): Il blasone dell'arcivescovo di Salerno Niccolò d'Ajello nelle illustrazioni di Pietro da Eboli. Bollettino storico di Salerno e Principato Citra. VII. nr. 1-2.51-60.

D’Aiello, Gaetano (1987): Il manoscritto del Prignano e le fonti nello studio della famiglia d'Ajello. Bollettino storico di Salerno e Principato Citra. V. nr. 1. 63-75.

D’Ajello, Gaetano (2003): Lo statista Matteo d'Ajello e 
la politica nazionale al tramonto della monarchia normanna di Sicilia. Annali storici di Principato Citra. I. nr. 1. (gennaio-giugno). 17-66.

D’Elia, Mario (2011): Uno scrigno sotto il moggio. Salerno, Stefano Taccone.

De Angelis, Michele (1933): Il sepolcro dei due Romualdo nella cattedrale di Salerno. Archivio storico della Provincia di Salerno. VI. fasc. IV (ottobre-dicembre). 313-321. [publication intégrale de la même revue, I. N.S. gennaiodicembre 1933].

De Angelis, Michele (1936): Il duomo di Salerno nella sua storia, nelle sue vicende e nei suoi monumenti. Salerno, Di Giacomo.

De Angelis, Michele (1937): Nuova guida del duomo di Salerno, Salerno, Di Giacomo.

De Crescenzo, Alfredo (1921): Il sepolcro dei due Romualdo. Archivio storico della Provincia di Salerno. I. nr. 4. (ottobre-dicembre) 337-342.

De Crescenzo, Alfredo. (1926): Le figurazioni di due sarcofagi pagani. Archivio storico della Provincia di Salerno. IV. nr. 4 (1926). 231-234.

De Cunzo, Mario (1988): Alfano I costruttore del Duomo di Salerno. Bollettino storico di Salerno e Principato Citra. VI. nr. 1. 6-10.

De Simone, Vincenzo (1990): Larcivescovo Federico Fregoso e il feudo di Montecorvino. Rassegna Storica Salernitana. N.s. VII. nr. 2. 211-220.

De Simone, Vincenzo (1991): L'ubicazione dell'antica cattedrale dei vescovi salernitani. Rassegna Storica Salernitana. N.s. VIII. nr. 1. 179-184.

Del Grosso, Maria Antonietta (1984): L'arcivescovo Marco Antonio Marsilio Colonna "protector et defensor » di Salerno. Bollettino storico di Salerno e Principato Citra. II. nr. 2. 49-53.

Del Grosso, Maria Antonietta (1996-1998): Gli arcivescovi salernitani. Un esempio di feudalità ecclesiastica nel sec. XVI. Bollettino storico di Salerno e Principato Citra. XIV-XVI. 79-176.

Delogu, Paolo - Peduto, Paolo (eds.): Salerno nel XII secolo: istituzioni, società, cultura. Atti di Convegno internazionale, Raito di Vietri sul mare 16/20 giugno 1999. Salerno, Incisivo.

FAlco, Giorgo (1912a): Sull'autenticità delle opere di Alfano, arcivescovo di Salerno (1058-1085). Bullettino dell'Istituto storico italiano per il Medioevo. V. nr. 32. 1-6.

FALCo, Giorgo (1912b): Un vescovo poeta del secolo XI. Alfano di Salerno. Archivio della Reale Società Romana di storia patria. XXXV. 439-481.
Fonseca, Cosimo Damiano (1996): Longobardia e Longobardi nell'Italia meridionale. Le istituzioni ecclesiastiche. In: ANDENNA - PICASso 1996.3-17.

Gaglione, Mario (2002): Sulla pretesa commissione dei monumenti sepolcrali durazzeschi in Napoli da parte di Margherita d'Angiò-Durazzo nel 1399 Napoli Nobilissima. seria V., III. nr. 3-4.113-133.

GALDI, AmALIA (1994): Un'altra questione di agiografia salernitana: i santi martiri Fortunato, Caio ed Ante. Rassegna storica salernitana. XI. nr. 1 (giugno). 7-37.

GALdi, Amalia (1996): Il santo e la città: il culto di s. Matteo a Salerno tra X e XVI secolo. Rassegna storica salernitana. XIII. nr. 1. (giugno). 21-92.

GALDI, AMALIA (1999): La diffusione del culto del santo patrono: l'esempio di s. Matteo di Salerno. In: Vitolo, Giovanni (ed.): Pellegrinaggi e itinerari dei santi nel Mezzogiorno medievale. Napoli, Liguori. 181-191. (Europa Mediterranea. Quaderni, 14.)

Galdi, Amalia (2000): Il calendario salernitano del 1434. Rassegna storica salernitana. XVII. nr. 1. (giugno). 95-158.

GaLdi, Amalia (2002-2003): Grammazio, un vescovo sanctae memoriae nella Salerno medievale. Schola Salernitana. Annali. VII-VIII. 10-26.

Galdi, Amalia (2003): Principi, vescovi e santi in Salerno longobarda. In: I Longobardi dei Ducati di Spoleto e di Benevento, atti del XVI Congresso internazionale di studi sull'alto medioevo, Spoleto, 20-23 ottobre 2002 - Benevento, 24-27 ottobre 2002. I-II. Spoleto, II. 1429-1449. (Atti dei Congressi, XVI.)

Galdi, Amalia (2004a): Santi, territori, poteri e uomini nella Campania Medievale. 2004. Salerno, Laveglia.

GALDI, AmAlia (2004b): I santi e la città. Agiografie e dedicazioni. In: Delogu - Peduto 2004. 170-187.

Galdi, Amalia (2011): Conflittualità, potere regio e dinamiche sociali nella Salerno angioina. Momenti di una ricerca in progress. Mélanges de l'École française de Rome. Moyen Âge. 123. nr. 1. 243-256.

Galdi, Amalia (2013): Il « manoscritto Pinto » come fonte storica per alcune famiglie di antica nobiltà (XI-XV secolo). In: CAPRIOLO, Giuliana - Cirillo, Giuseppe - Del Grosso, Maria Antonietta - DELLA Monica, Ugo - de Simone, Vincenzo - Galdi, Amalia: Specchi di nobiltà. Il manoscritto Pinto della Biblioteca Provinciale di Salerno. Salerno, Provincia di Salerno. 87-110. 
Gandolfo, Francesco (1984): La cattedra « gregoriana » di Salerno. Bollettino storico di Salerno e Principato Citra. II. nr. 4. 5-29.

Garms, Jörg - Juffinger, Roswita - Ward PerKINS, Bryan (ed.) (1981): Die mittelalterlichen Grabmäler in Rom und Latium von 13 bis zum 15 jahrhundert. 1. Band die Grabplatten und Tafeln. Wien, Verlag der Östereichischien Akademie der Wissenschaften.

GARMS, JÖRG - RoMANINI, ANgiola MARIA (ed.) (1990): Skulptur und Grabmal des Spätmittelalters in Rom und Italien. Akten des Kongresses "Scultura e monumento sepolcrale del tardo medioevo a Roma e in Italia » (Rom, 4.-6. Juli 1985). 1990. Wien, Verlag der Östereichischien Akademie der Wissenschaften.

GArms, Jörg - Sommerlechner, AndreA - Telesko, WERNER (ed.) (1994): Die mittelalterlichen Grabmäler in Rom und Latium von 13 bis zum 15. jahrhundert. 2. Band: Die Monumentalgräber. Wien, Verlag der Östereichischien Akademie der Wissenschaften.

Garufi, Carlo Alberto (ed.) (1922): Necrologio del Liber confratrum di S. Matteo di Salerno. Roma, Bottega d'Erasmo. (Fonti per la storia d'Italia pubblicate dall'Istituto storico italiano, [poi] pubblicate dall'Istituto storico italiano per il Medioevo, 56).

Garzillo, Fernando (1999): Un conteso giuspatronato nella cattedrale di Salerno. Rassegna storica salernitana. N.s. XXXI. 255-272.

Gentile, Egildo (1933): Pergamene dei monasteri di Salerno. Archivio storico per la provincia di Salerno. N.s. I. (gennaio-dicembre). 290-301.

Gentile, Egildo (1934): Pergamene di monasteri di Salerno nella prolusione al Corso di Paleografia del R. Archivio di Stato di Napoli. Archivio storico per la provincia di Salerno. N.s. II. nr. 1. (gennaio-marzo). 19-21.

Glass, Dorothy (2004): The Pulpits in the Cathedral at Salerno. In:DELOGU - PEDUTO 2004. 213-237.

Giordano, Anna (2014): Le pergamene dell'Archivio Diocesano di Salerno (841-1193). Battipaglia, Laveglia\&Carlone.

Granito, Gennaro (1984): Giovan Battista Prignano e i manoscritti salernitani della Biblioteca Angelica di Roma. Bollettino storico di Salerno e Principato Citra. II. nr. 1. 81-87.

Granito, Gennaro (1992): I Gesualdo dalla potenza normanna ai bagliori della fortuna angioina. Bollettino storico di Salerno e Principato Citra. X. nr. 1-2.37-45.
Grierson - Travaini (1998) : Medieval European Coinage. Italy (III) South Italy, Sicily, Sardinia,vol. 14. Cambridge, Cambridge University Press.

GUARIGLIA, RAFFAELE (1943): Un ambasciatore salernitano del sec. XV. Labate Ruggi. Rassegna Storica Salernitana. IV. nr. 1-2 (gennaio-giugno). 27-56.

Herklotz, INGo (2001): «Sepulcra » « Monumenta» del Medioevo. Studi sull'arte sepolcrale in Italia. Napoli, Liguori.

Hoffmann, Hartmut (ed.) (1980): Chronica Casinensis. Die Chronik von Montecassino. Hannoverae, Hahn. (MGH Scriptores, XXXIV)

Iguanez, Mauro - Mattei-Cerasoli, Leone - Sella, Pietro (ed.) (1942): Rationes decimarum Italiae nei secoli XII XXV. Campania. Città del Vaticano, Biblioteca Apostolica Vaticana.

IANNELli, Maria Antonietta (1992): Una fucina medievale a Salerno. Bollettino storico di Salerno e Principato Citra. X. nr. 1-2. 19-26.

JACQUART, DANIELLE - PARAVICINI BAgLiani, Agostino (ed.) (2007): La Scuola Medica Salernitana. Gli autori e i testi. Firenze, SISMEL - Edizioni del Galuzzo.

KAMP, Norbert (1978): Politica ecclesiastica e struttura sociale nel Regno svevo di Sicilia. Archivio storico per le Province napoletane. III s. XVI. 9-21.

Kehr, Paul Fridolin (1935): Italia Pontificia. VIII, Regnum Normannorum - Campania. Berlin, Weidmann.

Kronig, Wolfgang (1969): Il duomo normanno di Salerno nei disegni di Louis-Jean Desprez. Napoli Nobilissima. 8. 217-222.

LAMBert, Chiara (2013): I documenti epigrafici. In: PEDUTO - FioRILlo - CoROlla 2013. 45-59.

Marino, Roberto (1947-1948): L'atrio del Duomo di Salerno alla luce dei recenti restauri. Rassegna Storica Salernitana. VIII. nr. 1-4 (gennaio-dicembre) 110-114; IX.nr. 1-4 (gennaio-dicembre). 217-226.

Marsilio Colonna, Marco Antonio (1580): De vita et gestis Beati Matthaei Apostoli et Evangelistae. Napoli, Horatius Salvianus.

Mattei, Cerasoli M. (1920): Dialcuni vescovi poco noti. Archivio storico per le Provincie napoletane. N.s. IV, XLIII dell'intera collezione, 363-382 ; N.s. V, XLIV dell'intera collezione, 1-4. (maggio). 310-335.

Maurano, Attilio (1988): I restauri della cattedrale di Salerno dal 1900 al 1980. Bollettino storico di Salerno e Principato Citra. VI. nr. 1. 11-19.

Miccio, Gennaro (2011), Le vie dell'acqua. In : Cam- 
panelli, Adele (ed.): Dopo lo tsunami Salerno antica. Napoli, Arte'm. 336-341.

Morcaldi, Michele - Schiani, Mauro - De Stefano, Silvano (ed.) (1878), Codex Diplomaticus Cavensis. Mediolani - Pisis - Neapoli, Bibliopola.

Mosca, Gaspare (1594): De Salernitanae ecclesiae episcopi et archiepiscopis catalogus. Neapoli, ex typographia Stelliolae ad Portam Regalem.

Mosca, Gaspare (1930): De Salernitanae ecclesiae episcopi et archiepiscopis catalogus, nunc ab Arturo Capone ... omnibus archiepiscopis auctus qui ab a. MDXCI usque ad nostram aetatem Salernitanam ecclesiam rexerunt vulgatus. Subiaco, Tipografia dei monasteri.

Montaubin, Pascal (2003): "Avec de l'Italie qui descendrait l'Escault». Guido da Collemezzo, évêque de Cambrai (1296-1306). In: Barthélemy, Dominique - Martin, Jean-Marie (eds.) : Liber Largitorius. Études d'histoire médiévale offertes à Pierre Toubert par ses élèves. Genève - Paris, Droz. 477-502.

Nel X centenario della traslazione di S. Matteo a Salerno. 954-1954. Collana di studi commemorativi. [Salerno, Stabilimento tipografico fratelli Di Giacomo].

Oldoni, Massimo (2003): Guarna, Romualdo. In: Dizionario Biografico degli Italiani. Vol. 60. Roma, Istituto dell'Enciclopedia Italiana Treccani. 400-403.

Peduto, Paolo (2013): Consuetudine ed evoluzione dell'antico nelle costruzioni di Arechi II. In: PEDUTO - Fiorillo - Corolla 2013. 1-19.

Peduto, Paolo - Fiorillo, Rosa - Corolla, ANGELA (ed.) (2013): Salerno. Una sede ducale della Langobardia meridionale. Spoleto, Fondazione Centro Italiano di Studi Sull'alto Medioevo.

Romano Rosa (2003): La bottega durazzesca e la scultura napoletana nei decenni centra- li del XIV secolo. Arte Cristiana. XCI. 18-28. Romito, Matilde (2013): Le terme romane. In: Peduto - Fiorillo - Corolla 2013. 21-31.

Sangermano, Gerardo (2000): Poteri vescovili e signorie politiche nella Campania medievale. Lecce, Mario Congedo.

Savio, C. Fedele (1901-1902): I vescovi di Salerno nei secoli IX-X. Atti della R. Accademia delle Scienze di Torino. XXXVII. 104-113.

Schiavo, Armando (1945a): Note sul duomo di Salerno. Rassegna Storica Salernitana. VI. nr. 3-4. (luglio-dicembre). 241-243.

Schiavo, Armando (1958): L'architettura negli avori e ipotesi sulle loro origini. Rassegna Storica Salernitana. XIX. nr. 1-4. 75-86.

SPINELli, Giuseppe (1996): Il Papato e la riorganizzazione ecclesiastica della Longobardia meridionale. In: ANDENNA - PiCASSO 1996. 19-42.

Staibano Luigi (1871): Guida del duomo di Salerno composta ad uso de' viaggiatori. Salerno, Stabilimento Tipografico Nazionale.

Taviani Carozzi, Huguette (1991): La Principauté lombarde de Salerne $\left(\mathrm{IX}^{e}-\mathrm{XI}\right)$ siècle. Pouvoir et société en Italie Lombarde méridionale. I-II. Rome, École Française de Rome (Collection de l'École Française de Rome, 152).

Timpano, Francesco (1986): La sistemazione del sepolcro della regina Margherita di Durazzo nel Duomo di Salerno. Bollettino storico di Salerno e Principato Citra. IV. nr. 1. 67-71.

Vitolo, Giovanni (1999): Vescovi e diocesi. In: Galasso, Giuseppe - Romano, Rosario. (ed.): Storia del Mezzogiorno. III. Alto Medioevo. Napoli, Editalia. 75-101.

Westerbergh, Ulla (ed.) (1956): Chronicon Salernitanum. A critical edition with studies on literary and historical sources and on language. Stockholm, Almqvist \& Wiksell. 75-88. 